$\underline{\text { Preprint typeset in JHEP style - HYPER VERSION }}$

hep-th/0412188

AEI-2004-107

NSF-KITP-04-122

\title{
The Factorized S-Matrix of CFT/AdS
}

\author{
Matthias Staudacher \\ Max-Planck-Institut für Gravitationsphysik, Albert-Einstein-Institut* \\ Am Mühlenberg 1, D-14476 Potsdam, Germany \\ and \\ Kavli Institute for Theoretical Physics, University of California \\ Santa Barbara, CA 93106 USA \\ Email: matthias@aei.mpg.de
}

\begin{abstract}
We argue that the recently discovered integrability in the large- $N$ $\mathrm{CFT} / \mathrm{AdS}$ system is equivalent to diffractionless scattering of the corresponding hidden elementary excitations. This suggests that, perhaps, the key tool for finding the spectrum of this system is neither the gauge theory's dilatation operator nor the string sigma model's quantum Hamiltonian, but instead the respective factorized Smatrix. To illustrate the idea, we focus on the closed fermionic $\mathfrak{s u}(1 \mid 1)$ sector of the $\mathcal{N}=4$ gauge theory. We introduce a new technique, the perturbative asymptotic Bethe ansatz, and use it to extract this sector's three-loop S-matrix from Beisert's involved algebraic work on the three-loop $\mathfrak{s u}(2 \mid 3)$ sector. We then show that the current knowledge about semiclassical and near-plane-wave quantum strings in the $\mathfrak{s u}(2), \mathfrak{s u}(1 \mid 1)$ and $\mathfrak{s l}(2)$ sectors of $A d S_{5} \times S^{5}$ is fully consistent with the existence of a factorized S-matrix. Analyzing the available information, we find an intriguing relation between the three associated S-matrices. Assuming that the relation also holds in gauge theory, we derive the three-loop S-matrix of the $\mathfrak{s l}(2)$ sector even though this sector's dilatation operator is not yet known beyond one loop. The resulting Bethe ansatz reproduces the three-loop anomalous dimensions of twist-two operators recently conjectured by Kotikov, Lipatov, Onishchenko and Velizhanin, whose work is based on a highly complex QCD computation of Moch, Vermaseren and Vogt.
\end{abstract}

KEYwORDs: AdS-CFT Correspondence; Duality in Gauge Field Theories.

*Permanent Address 


\section{Contents}

1. Introduction and Conclusions 1

2. One-Loop Scattering in Planar $\mathcal{N}=4$

2.1 The $\mathfrak{s u}(2)$ Bosonic Sector 5

2.2 The $\mathfrak{s u}(1 \mid 1)$ Fermionic Sector 8

2.3 The $\mathfrak{s l}(2)$ Derivative Sector 10

2.4 Embedding of the Previous Sectors into $\mathfrak{s u}(2,2 \mid 4)$

3. Three-Loop S-Matrix for the $\mathcal{N}=4$ Fermionic Sector 14

4. S-Matrices for Quantum Strings at Large Tension 19

4.1 The $\mathfrak{s u}(2)$ Bosonic Sector 23

4.2 The $\mathfrak{s u}(1 \mid 1)$ Fermionic Sector 25

4.3 The $\mathfrak{s l}(2)$ Derivative Sector 26

5. Three-Loop S-Matrix for the $\mathcal{N}=4$ Derivative Sector 27

6. Summary and Musing 30

\section{Introduction and Conclusions}

There is mounting evidence that four-dimensional strictly planar $\mathcal{N}=4$ Yang-Mills gauge theory is integrable. Likewise, there are strong indications that free IIB superstring theory on the curved space $A d S_{5} \times S^{5}$ is also integrable. This means that there is hope that the spectrum of both theories might be exactly computable. If true, the AdS/CFT duality conjecture becomes falsifiable: Either the spectrum of the two models agrees, or it does not. This is good news, since, according to scientific tradition, falsifiability is an important feature of a theory, to be distinguished from creative speculation.

The first, crucial hint that conformal $\mathcal{N}=4$ gauge theory might be integrable in the planar limit was discovered in a beautiful paper by Minahan and Zarembo [1]. There it was shown that the spectrum of $\mathcal{N}=4$ conformal operators may be obtained by diagonalizing an integrable quantum spin chain. The observation was initially restricted to the subset of scalar operators at one loop. However, shortly after, strong evidence was found that integrability extends to higher loops [2], and, 
at least at one loop, to the full set of $\mathcal{N}=4$ operators [3]. Integrable structures have appeared before in planar QCD, starting with the pioneering work of Lipatov [四] (for a comprehensive review, see [5]). There it was always considered to be due to approximate, hidden symmetries, which can lead to useful information on high energy scattering and QCD anomalous dimensions. In $\mathcal{N}=4$ [1, 2, 3] the goal is more ambitious. One would like to exactly solve a four-dimensional gauge theory.

In a parallel, intertwined development integrable structures were also observed in the worldsheet theory of strings on $A d S_{5} \times S^{5}$. First, there were some hints that the relevant classical coset sigma model is integrable [6]. Later it was shown in detail how finite-dimensional reductions of the classical sigma model lead to integrable classical mechanics systems of Neumann type [7]. The reduction may be undone by the Bäcklund transformation, and the infinite tower of commuting charges of the original sigma model is recovered [8]. Subsequently, in an important paper by Kazakov, Marshakov, Minahan and Zarembo, it was demonstrated how the spectrum of finite-gap solutions of the classical sigma model may be obtained by the classical inverse scattering method [9]. In [10] first steps toward finding the quantum spectrum of the sigma model were taken, and an approximate S-matrix was proposed. Very recently, the Hamiltonian of the classical bosonic string propagating on $A d S_{5} \times S^{5}$ was shown to be integrable by constructing, in a special gauge, the corresponding Lax representation [11].

How should one proceed toward, for one, exactly solving the planar "CFT", namely the large $N$ limit of $\mathcal{N}=4$ gauge theory, and, secondly, the worldsheet theory of strings on $A d S_{5} \times S^{5}$ ? Let us assume, as a working hypothesis, that both models are indeed not only approximately, but in fact completely integrable. If true, proceeding with the construction of the gauge theory's dilatation operator, loopby-loop, or meticulously quantizing the full sigma model, including fermions, might prove too hard. In fact, integrable systems do not always have simple Hamiltonians. To the contrary, the Hamiltonian might be quite intricate in order to realize the subtle hidden symmetries responsible for integrability.

Is there a simpler object which encodes the spectrum of an integrable quantum system? In fact there is: The S-Matrix. Quantum integrability is deeply tied to the concept of diffractionless, factorized scattering ${ }^{1}$. It means that the elementary excitations of a quantum many-body system interact only through a sequence of two-body scattering processes which may lead to the exchange of quantum numbers and momenta, but do not alter the magnitudes of the latter. This is the next-best

\footnotetext{
${ }^{1}$ We are discussing in this paper an internal S-Matrix describing the scattering of elementary excitations on a lattice hidden inside the trace of of gauge invariant composite local operators. It should not be confused with the external S-matrix of $\mathcal{N}=4$ which refers to multi-gluon amplitudes in four-dimensional space time. Recently dramatic progress was also achieved in this direction, see [12], and references therein and thereafter. It would be exciting if a relation between the internal and the external S-matrix could be found.
} 
thing to a free system! Interaction does take place, but scattering only leads to a permutation of a fixed set of momenta (and, possibly, of quantum numbers).

Here we would like to propose that one should try to directly construct the Smatrix of integrable gauge and string theory. Hopefully, the two will then agree. Unfortunately we do not have a very concrete proposal about how to go about it in a direct fashion. However, since the AdS/CFT system possesses a huge amount of symmetry, and, in particular, apparently an infinite number of hidden conserved charges, we may certainly hope that methods to "bootstrap" the S-matrix will eventually be found.

As a more modest first step, we will analyze part of what is known about the gauge theory's dilatation operator, and about the spectrum of AdS strings, in order to get a glimpse at how the S-matrix might look like. We think that the results are encouraging. For simplicity we will restrict ourselves, in this paper, to three simple two-component sectors of the full superconformal, $\mathfrak{p s u}(2,2 \mid 4)$ symmetric, system. These are a compact $\mathfrak{s u}(2)$ subsector (two bosons), a compact $\mathfrak{s u}(1 \mid 1)$ subsector (one boson, one fermion), and a non-compact $\mathfrak{s l}(2)$ subsector (two bosons).

Let us recall what is currently known about the $\mathcal{N}=4$ gauge theory's dilatation operator and its Bethe ansatz. The latter may be constructed once the S-matrix is known. At one loop, the dilatation operator was derived for the complete set of $\mathfrak{p} \mathfrak{s u}(2,2 \mid 4)$ operators [13]. It was shown to be integrable in [3], and the associated Bethe ansatz was constructed. Beyond one loop, we have Beisert's two- and three loop dilatation operator in the "maximally compact" subsector $\mathfrak{s u}(2 \mid 3)$ [14]. This includes the $\mathfrak{s u}(2)$ (here it was initially found in [2]) and $\mathfrak{s u}(1 \mid 1)$ sectors, but not the $\mathfrak{s l}(2)$ sector. No two- and three loop Bethe ansatz is known, except for the $\mathfrak{s u}(2)$ sector, where it was constructed in [15]. The $\mathfrak{s u}(2)$ dilatation operator was then extended to five loops, assuming integrability as well as BMN scaling. A fiveloop Bethe ansatz was found experimentally, and an all-loop (asymptotic) Bethe ansatz was proposed [16]. For a closely related system, see [17. Note that the three-loop predictions of the three-loop dilatation operator in the $\mathfrak{s u}(2)$ sector were recently spectacularly confirmed, for two states, by a full-fledged, rigorous field theory computation [18]. For a detailed review on most aspects concerning the $\mathcal{N}=4$ dilatation operator, see [19].

One thing to note about the higher loop dilatation operators [2, 14, 16] is that they are very complicated, and appear to increase exponentially in complexity as the loop order increases (see e.g. appendix A.1 in [16]). In fact, the $\mathfrak{s u}(2 \mid 3)$ dilatation operator [14] is only known in "algorithmic" form.

In contradistinction, the S-matrices appear to allow for a much simpler description. E.g. the (asymptotic) S-matrix of [16] may be written in a very compact fashion ( $c f$ (4.26) with (4.27)). We shall see another example in this paper, where we extract the three-loop S-matrix of the $\mathfrak{s u}(1 \mid 1)$ subsector from [14]. This requires designing a new technique which one might term PABA: Perturbative Asymptotic 
Bethe Ansatz. To motivate it, we derive in some, hopefully pedagogical, detail various one-loop Bethe ansätze at the beginning of the paper. The final result for $\mathfrak{s u}(1 \mid 1)$ is rather compact, while the "source", namely the two- and three-loop dilatation operator, fills at least a page. Clearly things will get worse for the operator at even higher loops, while we suspect the existence of a simple all-loop expression for the S-matrix.

In light of the AdS/CFT correspondence it is very natural to ask about the Smatrix of the string sigma model. However, the sigma model believed to describe strings on $A d S_{5} \times S^{5}$ is far more complicated than those theories where S-matrices have already been found. Still, one could try to look for similar models in order to derive some hints, which is an approach that has recently been followed in [20].

Alternatively, one can attempt to derive useful clues from available information on the sigma model's spectrum. It comes from two sources. We already mentioned [9], where an equation describing the classical spectrum in the $\mathfrak{s u}(2)$ sector (in gauge theory connotation) was derived. Recently, the procedure was also applied to the $\mathfrak{s l}(2)$ sector [21]. It is found that the most general classical finite-gap solution may be described by an algebraic curve. But does it contain information on the S-matrix, which is an inherently quantum concept? Here it turns out that the crucial connection does not come from the curve as such, but from the equation describing it. Namely, the equation may be brought into a "scattering form" which allows to draw conclusions about the interactions of the quantum excitations as one approaches the classical limit [16], [10]. The crucial intuition for finding the correct interpretation comes from the fact that at one- and two-loops the classical string sigma model behaves very similarly [22, 7] (but, starting from three loops, not identical [15]) to the gauge theory when the associated operators become very "long". On the gauge side one finds rather similar equations in this "thermodynamic" situation [23, 24], [15]. Since the latter, which are derived from the discrete Bethe equations, do have a scattering interpretation, the detailed comparison allows one to also bring the string equations into "scattering form". This logic gave a hint about the string's S-matrix in the $\mathfrak{s u}(2)$ sector [10], and we will apply it below, using [21], to learn about the S-matrix in the $\mathfrak{s l}(2)$ sector.

A second important source of spectral information comes from the plane wave limit of strings on $A d S_{5} \times S^{5}$, where the worldsheet theory becomes free, and may be thus be quantized exactly [25]. Much intuition about the nature of the elementary excitations in this limit comes from subsequently comparing to gauge theory, i.e. from considering the famous BMN limit [26]. Recent studies of the near-BMN limit [27, 28, 29, 30, 31] yield information on how these excitations begin to interact. As we will show below, these interactions are fully consistent with the idea of an underlying factorized S-matrix. In fact, we will use the results of [29, 30, 31 to derive part of the string S-matrix in the fermionic sector $\mathfrak{s u}(1 \mid 1)$. This is important, as it is unclear what may be learned from the classical sigma model for this sector. (For some recent 
work in this direction, see [32].) After all, fermions are inherently quantum. They will actually serve as a "missing link" in the present story ...

... which finishes as follows. Comparing the approximate S-matrices extracted from string theory in the three sectors $\mathfrak{s u}(2), \mathfrak{s u}(1 \mid 1)$ and $\mathfrak{s l}(2)$, we find a simple equation relating them. We then apply the relation to three-loop gauge theory, and write down the S-matrix in the $\mathfrak{s l}(2)$ sector, even though we do not know the higher loop dilatation operator. The resulting spectrum is then successfully checked in the case of two-impurity states, where the spectrum is known from superconformal symmetry 33. More excitingly, we may compare to recent results of Kotikov, Lipatov, Onishchenko and Velizhanin [35], who proposed three-loop exact anomalous dimensions for $\mathcal{N}=4$ twist-two operators. These dimensions were first found at one-loop from the OPE of four-point functions in [37], and the first few, at two loops, in [38]. This author does not understand the derivation of Kotikov et.al., but it is clear that the crucial input is a computationally highly intensive three-loop QCD field theory computation recently completed after a many-year effort by Moch, Vermaseren and Vogt [36] (see also the comments made in [12]). It involved the evaluation of $\mathcal{O}\left(10^{6}\right)$ auxiliary integrals, and the development of cutting-edge algorithmic techniques. The spectrum of twist-two operators derived from our S-matrix agrees with the results of Kotikov et.al.

Can we do better and find the full S-matrix of the CFT and strings on AdS in a more direct fashion? Note that this possibility entirely hinges on whether integrability is really exact in either theory. It does not necessarily depend on whether the AdS/CFT duality [39] is actually valid dynamically. We feel that the present work demonstrates once again that it is surely useful.

\section{One-Loop Scattering in Planar $\mathcal{N}=4$}

\subsection{The $\mathfrak{s u}(2)$ Bosonic Sector}

This sector consists of operators of the type

$$
\operatorname{Tr} \phi^{M} Z^{L-M}+\ldots=\operatorname{Tr} \phi^{M} Z^{J}+\ldots,
$$

where $J$ denotes an R-charge w.r.t. $\mathrm{SO}(6)$ and $M$ the number of "impurities". The partons $Z$ and $\phi$ are two out of the three complex adjoint scalars of the $\mathcal{N}=4$ model. The dots indicate that we need to consider all possible orderings of them inside the trace, and diagonalize the set of such operators with respect to dilatation. This is most easily done when interpreting the dilatation operator as a spin chain Hamiltonian [1]. In the spin chain interpretation $L$ is the chain length and $M$ the number of excitations. It is convenient to open up the trace and replace it by a quantum mechanical state on a one dimensional lattice of $L$ sites:

$$
\operatorname{Tr}(\phi Z Z \phi \ldots \phi Z) \rightarrow|\phi Z Z \phi \ldots \phi Z\rangle
$$


Let us label the sites of this lattice by a discrete coordinate $x$. (So in the example of (2.2) we see that we have a $\phi$ at $x=1,4, L-1$ and a $Z$ at $x=2,3, L)$. The fact that we originally had a trace leads to two consequences, to be distinguished. The first is that, since the trace links the matrix indices of the first and last parton, the chain has periodic boundary conditions: $x=L+1$ is to be identified with $x=1$. The second is that the cyclicity of the trace requires us to project onto states whose total lattice momentum is zero, see (2.12) below.

The planar one-loop position space Hamiltonian reads

$$
H_{0}=\sum_{x=1}^{L}\left(1-\mathcal{P}_{x, x+1}\right)=\sum_{x=1}^{L} \frac{1}{2}\left(1-\vec{\sigma}_{x} \cdot \vec{\sigma}_{x+1}\right),
$$

and may be expressed with the help of the permutation operator $\mathcal{P}_{x, x+1}$ which exchanges the partons at sites $x$ and $x+1$, as first noticed in [1, 40]. It may alternatively be interpreted as an $\mathfrak{s u}(2)$ nearest neighbor spin chain [1] if we say that $Z$ is a spin up with 2 -spinor $(1,0)$ and $\phi$ is a spin down with 2 -spinor $(0,1)$. Then the spin operator at lattice site $x$ contains the three Pauli matrices $\vec{\sigma}_{x}=\left(\sigma_{x}^{1}, \sigma_{x}^{2}, \sigma_{x}^{3}\right)$ and the Hamiltonian may be written in the the form of a sum over nearest neighbor spin-spin interactions.

The two-body states are defined by

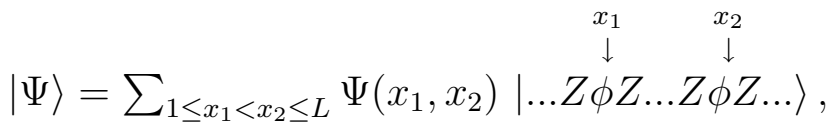

where $x_{1,2}$ (with $x_{1}<x_{2}$ ) label the positions of the two $\phi$ particles in the background of the $Z$ particles. In position space the Schrödinger equation $H_{0} \cdot|\Psi\rangle=E_{0}|\Psi\rangle$ becomes

$$
\begin{aligned}
& \text { for } \quad x_{2}>x_{1}+1 \text { : } \\
& E_{0} \Psi\left(x_{1}, x_{2}\right)=2 \Psi\left(x_{1}, x_{2}\right)-\Psi\left(x_{1}-1, x_{2}\right)-\Psi\left(x_{1}+1, x_{2}\right)+ \\
& +2 \Psi\left(x_{1}, x_{2}\right)-\Psi\left(x_{1}, x_{2}-1\right)-\Psi\left(x_{1}, x_{2}+1\right), \\
& \text { for } x_{2}=x_{1}+1 \text { : } \\
& E_{0} \Psi\left(x_{1}, x_{2}\right)=2 \Psi\left(x_{1}, x_{2}\right)-\Psi\left(x_{1}-1, x_{2}\right)-\Psi\left(x_{1}, x_{2}+1\right) .
\end{aligned}
$$

This difference equation is easily solved by Bethe's ansatz [42] for the position space wave function $\Psi\left(x_{1}, x_{2}\right)$ which reads

$$
\Psi\left(x_{1}, x_{2}\right)=e^{i p_{1} x_{1}+i p_{2} x_{2}}+S\left(p_{2}, p_{1}\right) e^{i p_{2} x_{1}+i p_{1} x_{2}} .
$$

It is based on the intuition that the partons should freely evolve down the trace with fixed momenta $p_{1}, p_{2}$ until they hit each other at $x_{2}=x_{1}+1$. If the system is integrable they should then simply either pass through each other, or else exchange momenta, 
with an amplitude given by the S-matrix $S\left(p_{1}, p_{2}\right)$. This scattering process is nondiffractive if the individual momenta $p_{k}$ are individually conserved. If we were to stay with the two-body problem this would of course always be true because of total momentum conservation. The miracle of integrability is that it remains true for the $M$-body problem. Put differently, we cannot prove integrability by only considering the two-body problem, but we can certainly find the S-matrix if we assume (or know) that the system is integrable.

Plugging the ansatz (2.7) into the Schrödinger equation (2.5),(2.6) one finds that it is indeed satisfied if, firstly, the energy is given by the dispersion law

$$
E_{0}=\sum_{k=1}^{M} 4 \sin ^{2}\left(\frac{p_{k}}{2}\right)
$$

with $M=2$, and, secondly, if the S-matrix is given by

$$
S_{\mathfrak{s u}(2)}\left(p_{1}, p_{2}\right)=-\frac{e^{i p_{1}+i p_{2}}-2 e^{i p_{1}}+1}{e^{i p_{1}+i p_{2}}-2 e^{i p_{2}}+1} .
$$

In line with intuition, the dispersion law (2.8) follows from the "generic" situation (2.5) while the S-matrix is deduced from the "colliding" situation (2.6).

All this is true for arbitrary values of the momenta $p_{k}$. As always in quantum mechanics the eigenvalues get fixed through the boundary conditions. Imposition of periodic boundary conditions $\Psi\left(x_{1}, x_{2}\right)=\Psi\left(x_{2}, x_{1}+L\right)$ on the wave function (2.7) leads to Bethe's equations:

$$
e^{i p_{1} L}=S\left(p_{1}, p_{2}\right) \quad \text { and } \quad e^{i p_{2} L}=S\left(p_{2}, p_{1}\right) .
$$

Their solution leads in many cases to complex values of the momenta $p_{k}$. This simply reflects the fact that the partons are not freely propagating, but mutually interacting inside a finite volume. In particular, they can form bound states.

The principle of non-diffractive scattering, if applicable, now allows us to take a big leap and immediately solve the $M$-body problem. The total phase factor acquired by a parton circling around the trace should simply be given as a product of the phase factors due to individual collisions with all other $M-1$ partons. The Bethe equations become

$$
e^{i p_{k} L}=\prod_{\substack{j=1 \\ j \neq k}}^{M} S\left(p_{k}, p_{j}\right), \quad k=1, \ldots, M
$$

with the same two-body S-matrix (2.9)! The total energy is of course still given by the sum over all $M$ local dispersion relations (2.8).

In our gauge theory application we have to take into account the fact that the trace is cyclic, which means that we are only interested in the zero-momentum sector:

$$
\sum_{k=1}^{M} p_{k}=0 \text {. }
$$


The one-loop anomalous dimension $\Delta$ of the original gauge theory operators (2.1) is then related to the energy spectrum of the zero-momentum states of the nearestneighbor spin chain through

$$
\Delta=\Delta_{0}+g^{2} E_{0}+\mathcal{O}\left(g^{4}\right), \quad \text { with } \quad g^{2}=\frac{g_{\mathrm{YM}}^{2} N}{8 \pi^{2}},
$$

where in the present case the classical scaling dimension is $\Delta_{0}=L$.

Finally, one commonly expresses the $\mathfrak{s u}(2)$ Bethe equations in a form familiar from the algebraic Bethe ansatz [41], as in [1, 3]. Changing variables by introducing

the so-called Bethe roots $u_{k}=\frac{1}{2} \cot \left(\frac{p_{k}}{2}\right)$, (2.11) becomes, after rewriting the S-matrix $(2.9)$,

$$
\left(\frac{u_{k}+\frac{i}{2}}{u_{k}-\frac{i}{2}}\right)^{L}=\prod_{\substack{j=1 \\ j \neq k}}^{M} \frac{u_{k}-u_{j}+i}{u_{k}-u_{j}-i}, \quad k=1, \ldots, M
$$

while the momentum constraint (2.12) and the energy (2.8) turn into

$$
\prod_{k=1}^{M} \frac{u_{k}+\frac{i}{2}}{u_{k}-\frac{i}{2}}=1 \quad \text { and } \quad E_{0}=\sum_{k=1}^{M} \frac{1}{u_{k}^{2}+\frac{1}{4}} .
$$

\subsection{The $\mathfrak{s u}(1 \mid 1)$ Fermionic Sector}

This sector consists of operators of the type

$$
\operatorname{Tr} \psi^{M} Z^{L-M}+\ldots=\operatorname{Tr} \psi^{M} Z^{J-\frac{M}{2}}+\ldots
$$

where $J$ denotes an R-charge w.r.t. $\mathrm{SO}(6)$ and $M$ the number of "impurities". The partons are for one a complex adjoint scalars $Z$, and secondly an adjoint gaugino $\psi$ (in $\mathcal{N}=1$ connotation). In the spin chain interpretation $L$ is the chain length and $M$ the number of excitations.

The planar one-loop Hamiltonian reads

$$
H_{0}=\sum_{x=1}^{L}\left(1-\Pi_{x, x+1}\right)
$$

It may be extracted from the complete one-loop $\mathcal{N}=4$ dilatation operator 113 and rewritten with the help of the graded permutation operator $\Pi_{x, x+1}$ which exchanges the partons at sites $x$ and $x+1$, picking up a minus sign if the exchange involves two fermions $\psi$. It was noticed in [30] that this Hamiltonian corresponds to a free lattice fermion. Here we would like to put this observation into a familiar condensed matter context. Let us rewrite the Hamiltonian in spin chain form by expressing it with the help of the three Pauli matrices $\sigma_{x}^{1}, \sigma_{x}^{2}, \sigma_{x}^{3}$ :

$$
H_{0}=\sum_{x=1}^{L}\left(\left(1-\sigma_{x}^{3}\right)-\frac{1}{2}\left(\sigma_{x}^{1} \sigma_{x+1}^{1}+\sigma_{x}^{2} \sigma_{x+1}^{2}\right)\right) .
$$


In spin chain language the bosonic partons $Z$ are "spin up" spinors $(1,0)$ and the fermionic partons $\psi$ are "spin down" spinors $(0,1)$. We now observe that (2.18) is the Hamiltonian of an XY spin chain in a magnetic field, which is well known to correspond to free lattice fermions. It is therefore even simpler than the XXX Heisenberg model! (The model is isotropic in the $\sigma_{x}^{1}, \sigma_{x}^{2}$ plane, so we could call it an XX spin chain.)

The two-body states are defined by

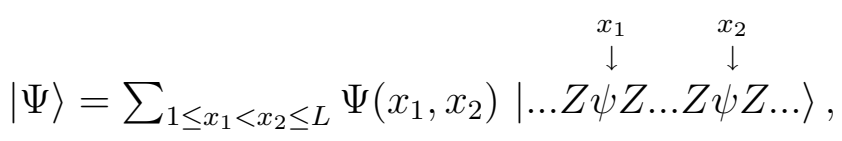

where $x_{1}, x_{2}$ label the positions of the two gauginos in the background of the bosonic $Z$ particles. In position space the Schrödinger equation $H_{0} \cdot|\Psi\rangle=E_{0}|\Psi\rangle$ becomes

$$
\begin{aligned}
& \text { for } x_{2}>x_{1}+1 \text { : } \\
& E_{0} \Psi\left(x_{1}, x_{2}\right)=2 \Psi\left(x_{1}, x_{2}\right)-\Psi\left(x_{1}-1, x_{2}\right)-\Psi\left(x_{1}+1, x_{2}\right)+ \\
& +2 \Psi\left(x_{1}, x_{2}\right)-\Psi\left(x_{1}, x_{2}-1\right)-\Psi\left(x_{1}, x_{2}+1\right), \\
& \text { for } x_{2}=x_{1}+1 \text { : } \\
& E_{0} \Psi\left(x_{1}, x_{2}\right)=4 \Psi\left(x_{1}, x_{2}\right)-\Psi\left(x_{1}-1, x_{2}\right)-\Psi\left(x_{1}, x_{2}+1\right) .
\end{aligned}
$$

These equations are identical to the ones of the $\mathfrak{s u}(2)$ model except for the innocent looking replacement of a factor of 2 by a 4 when comparing (2.6) and (2.21).

We now make the same Bethe ansatz (2.7) as in the case of the $\mathfrak{s u}(2)$ sector, and plug it into the difference Schrödinger equations (2.20), (2.21). The energy eigenvalue (2.8) remains unchanged, but the result for the S-matrix is very different:

$$
S_{\mathfrak{s u}(1 \mid 1)}\left(p_{1}, p_{2}\right)=-1
$$

So indeed the excitations behave as free fermions, and the S-matrix (2.22) reflects free fermi statistics. In fact, here the wavefunction (2.7) is simply a two-body Slater determinant. Some care has to be taken with Fermi statistics when imposing periodic boundary conditions; we now have $\Psi\left(x_{1}, x_{2}\right)=-\Psi\left(x_{2}, x_{1}+L\right)$. The "Bethe equations" are then:

$$
e^{i p_{1} L}=-S\left(p_{1}, p_{2}\right)=1 \quad \text { and } \quad e^{i p_{2} L}=-S\left(p_{2}, p_{1}\right)=1 .
$$

Due to integrability we can again immediately solve the one-loop $M$-body problem. Here there simply is no scattering at all, but we need to be careful with Fermi statistics. The $M$-body equations read

$$
e^{i p_{k} L}=1, \quad k=1, \ldots, M .
$$

These are of course immediately solved

$$
p_{k}=\frac{2 \pi n_{k}}{L},
$$


but due to fermi statistics the integer mode number $n_{k}$ are required to be all distinct. (The wavefunction is an $M \times M$ Slater determinant and vanishes if two momenta coincide). They also have to be restricted to the fundamental Brillouin zone in order to avoid overcounting the states. Furthermore, in gauge theory we are again only interested in the zero-momentum sector $\sum_{k=1}^{M} p_{k}=0$, which immediately translates into $\sum_{k=1}^{M} n_{k}=0$.

The one-loop anomalous dimension $\Delta$ of the original gauge theory operators (2.16) is still given by (2.13) where the classical scaling dimension is $\Delta_{0}=L+\frac{1}{2} M$.

\subsection{The $\mathfrak{s l}(2)$ Derivative Sector}

This sector consists of operators of the type

$$
\operatorname{Tr} D^{M} Z^{L}+\ldots=\operatorname{Tr} D^{S} Z^{J}+\ldots,
$$

where $J$ denotes an R-charge w.r.t. $\mathrm{SO}(6)$ and $M=S$ the number of "impurities" which corresponds to one of the two spin quantum numbers ${ }^{2} S$ of $\mathrm{SO}(2,4)$. The partons are for one a complex adjoint scalar $Z$, and secondly an adjoint lightcone covariant derivative $D$. The dots indicate that we need to consider all possible distributions of the covariant derivatives $D$ onto the scalars $Z$ inside the trace, and diagonalize the set of such operators with respect to dilatation. In the spin chain interpretation $L=J$ is the chain length and $M$ the number of excitations. Note that, unlike the previous two cases, the excitations do not contribute to the length of the spin chain. In particular, the number of excitations may exceed the total length of the chain. In this context, the length is also commonly called "twist". A "twist-two" operator is thus a very short spin chain of length 2 .

The one-loop Hamiltonian for this sector has a global $\mathfrak{s l}(2)$ invariance. It is nonpolynomial, and has been derived and described in [13]. In the planar limit it may be considered as an integrable nearest neighbor $\mathfrak{s l}(2)$ spin $-\frac{1}{2}$ spin chain [3]. Let us express the Hamiltonian $H$ through the Hamiltonian density $\mathcal{H}$ :

$$
H_{0}=\sum_{x=1}^{L} \mathcal{H}_{x, x+1} \text {. }
$$

Denoting partons with $0,1,2$ derivatives, i.e. $Z, D Z, D^{2} Z$ by, respectively, $|0\rangle,|1\rangle$, $|2\rangle$, we can read off the action of the Hamiltonian density on a neighboring pair of partons at lattice sites $x, x+1$ from [13] as

$$
\begin{gathered}
\mathcal{H} \cdot|1,0\rangle=|1,0\rangle-|0,1\rangle \quad \mathcal{H} \cdot|0,1\rangle=|0,1\rangle-|1,0\rangle \\
\mathcal{H} \cdot|1,1\rangle=2|1,1\rangle-|2,0\rangle-|0,2\rangle \\
\mathcal{H} \cdot|2,0\rangle=\frac{3}{2}|2,0\rangle-|1,1\rangle-\frac{1}{2}|0,2\rangle \quad \mathcal{H} \cdot|0,2\rangle=\frac{3}{2}|0,2\rangle-|1,1\rangle-\frac{1}{2}|2,0\rangle,
\end{gathered}
$$

${ }^{2}$ We will mostly avoid using the customary letter $S$ for the AdS spin in this paper in order to prevent, however unlikely, confusions with the S-matrix. Instead, we will use $M$ (for magnon number). 
where we have abbreviated $\mathcal{H}_{x, x+1}$ by $\mathcal{H}$. We have not written any terms beyond the two-body interactions as we shall not need them for finding the S-matrix. They are of course important for proving the factorizability of the many-body S-matrix, but not for finding it once we know (or believe) that the model is integrable. This observation might be very useful when constructing the Bethe ansatz for this and other sectors at higher loops, as it should apply there as well. It also illustrates nicely one of the main points of this paper, namely that the S-matrix is a much simpler object than the dilatation operator!

The two-body states are defined by

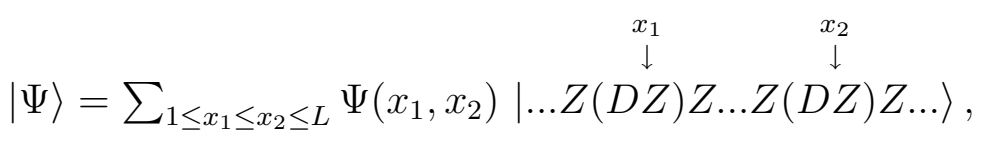

where $x_{1}, x_{2}$ label the positions of the two derivatives in the background of the bosonic $Z$ particles. Notice the following subtle difference as compared to (2.4), (2.19). The "particles" $D$ are not occupying their own lattice sites, but sit instead on top of the inert particles (=holes) $Z$. In particular, there may be multiple-occupancy, thus now $x_{1}=x_{2}$ is an allowed configuration! In condensed matter language we would say that the scattering is not "hard-core".

In position space the Schrödinger equation $H_{0} \cdot|\Psi\rangle=E_{0}|\Psi\rangle$ becomes

$$
\begin{aligned}
& \text { for } \begin{aligned}
& x_{2}>x_{1}: \\
& E_{0} \Psi\left(x_{1}, x_{2}\right)= 2 \Psi\left(x_{1}, x_{2}\right)-\Psi\left(x_{1}-1, x_{2}\right)-\Psi\left(x_{1}+1, x_{2}\right)+ \\
&+2 \Psi\left(x_{1}, x_{2}\right)-\Psi\left(x_{1}, x_{2}-1\right)-\Psi\left(x_{1}, x_{2}+1\right), \\
& \text { for } \quad x_{2}=x_{1}: \\
& E_{0} \Psi\left(x_{1}, x_{2}\right)= \frac{3}{2} \Psi\left(x_{1}, x_{2}\right)-\Psi\left(x_{1}-1, x_{2}\right)-\frac{1}{2} \Psi\left(x_{1}-1, x_{2}-1\right)+ \\
&+\frac{3}{2} \Psi\left(x_{1}, x_{2}\right)-\Psi\left(x_{1}, x_{2}+1\right)-\frac{1}{2} \Psi\left(x_{1}+1, x_{2}+1\right) .
\end{aligned}
\end{aligned}
$$

Notice that the first of these equations, (2.30), is valid for both the "generic" situation $x_{2}>x_{1}+1$ as well as for the nearest neighbor situation $x_{2}=x_{1}+1$. The second expression describes the ultralocal on-site interaction $x_{2}=x_{1}$ of the partons.

We make the same Bethe ansatz (2.7) as in the previous two cases. Plugging it into the first of the two Schrödinger difference equations (2.30) gives as before the energy eigenvalue (2.8), while the equation (2.31), describing the collision, yields the S-matrix :

$$
S_{\mathfrak{s l}(2)}\left(p_{1}, p_{2}\right)=-\frac{e^{i p_{1}+i p_{2}}-2 e^{i p_{2}}+1}{e^{i p_{1}+i p_{2}}-2 e^{i p_{1}}+1} .
$$

Notice that it differs from the $\mathfrak{s u}(2)$ case by the exchange $p_{1} \leftrightarrow p_{2}$. As before, the imposition of periodic boundary conditions, together with the principle of diffractionless scattering, leads to the Bethe equations of the form (2.11). 
Changing once again variables from the momenta $p_{k}$ to the Bethe roots $u_{k}=$ $\left.\frac{1}{2} \cot \left(\frac{p_{k}}{2}\right), 2.11\right)$ becomes

$$
\left(\frac{u_{k}+\frac{i}{2}}{u_{k}-\frac{i}{2}}\right)^{L}=\prod_{\substack{j=1 \\ j \neq k}}^{M} \frac{u_{k}-u_{j}-i}{u_{k}-u_{j}+i}, \quad k=1, \ldots, M
$$

while the momentum constraint and the energy are given as in the $\mathfrak{s u}(2)$ case by (2.15).

The one-loop anomalous dimension $\Delta$ of the original gauge theory operators (2.26) is again given by (2.13) where now the classical scaling dimension is $\Delta_{0}=$ $L+M$.

\subsection{Embedding of the Previous Sectors into $\mathfrak{s u}(2,2 \mid 4)$}

In the previous sections we demonstrated how to find the S-matrix of the three simplest two-component sectors of the superspin chain of [3] from the key principle of non-diffractive scattering. In the next chapter we will show, in the concrete example of the fermionic sector $\mathfrak{s u}(1 \mid 1)$, how to extend the method to higher loops. This requires the introduction of a new technique (the perturbative asymptotic Bethe ansatz). The method may also be applied to more than two components, but gets considerably more involved, as one needs to apply the so-called nested Bethe ansatz [43]. This is beyond the scope of the present paper. However, here we would like to show how, at one-loop, the above three two-component sectors are recovered as special cases embedded into the complete $\mathfrak{s u}(2,2 \mid 4)$ one-loop Bethe ansatz of [3].

In the complete super spin chain we have seven types of roots. It is useful to visualize them with the help of a $\mathfrak{s u}(2,2 \mid 4)$ Dynkin diagram. There are several possible choices, and here we will consider the "Beauty" version of [3]:

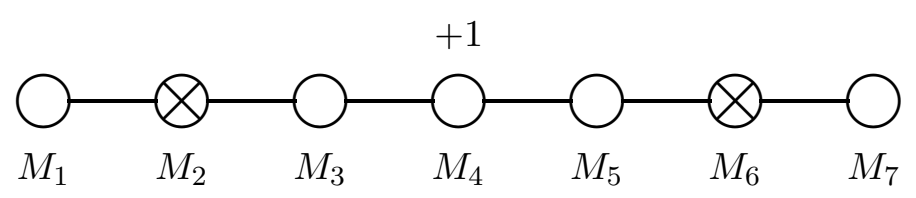

On top of the Dynkin diagram we have indicated the Dynkin labels of the representation corresponding to the vacuum state of the "Beauty" description (which corresponds to declaring the complex scalars $Z$ to be empty lattice sites or "holes"), and on the bottom the number $M_{k}$ of roots corresponding to the respective node. This way the vacuum state of a length $L$ chain is BPS. The full equations, which can be found in [3], may be concisely written using the Cartan matrix corresponding to this Dynkin diagram.

The $\mathfrak{s u}(2) M$-magnon sector is of course immediately obtained by only exciting the central node: $M_{k}=\delta_{k 4} M$. Now, for the fermionic $\mathfrak{s u}(1 \mid 1)$ chain of section 2.2 is 
not hard to verify [3] that it corresponds to the excitation pattern

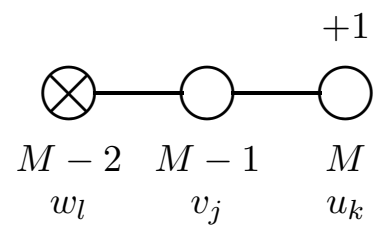

where we have omitted nodes that do not carry excited roots, and have introduced the notation $u_{k}, v_{j}$ and $w_{l}$ for the three types of roots. The Bethe equations of [3] become

$$
\begin{aligned}
\left(\frac{u_{k}+\frac{i}{2}}{u_{k}-\frac{i}{2}}\right)^{L} & =\prod_{\substack{k^{\prime}=1 \\
k^{\prime} \neq k}}^{M} \frac{u_{k}-u_{k^{\prime}}+i}{u_{k}-u_{k^{\prime}}-i} \prod_{j=1}^{M-1} \frac{u_{k}-v_{j}-\frac{i}{2}}{u_{k}-v_{j}+\frac{i}{2}} \quad 1 \leq k \leq M, \\
1 & =\prod_{k=1}^{M} \frac{v_{j}-u_{k}-\frac{i}{2}}{v_{j}-u_{k}+\frac{i}{2}} \prod_{\substack{j^{\prime}=1 \\
j^{\prime} \neq j}}^{M-1} \frac{v_{j}-v_{j^{\prime}}+i}{v_{j}-v_{j^{\prime}}-i} \prod_{l=1}^{M-2} \frac{v_{j}-w_{l}-\frac{i}{2}}{v_{j}-w_{l}+\frac{i}{2}} \quad 1 \leq j \leq M-1, \\
1 & =\prod_{j=1}^{M-1} \frac{w_{l}-v_{j}-\frac{i}{2}}{w_{l}-v_{j}+\frac{i}{2}} \quad 1 \leq l \leq M-2 .
\end{aligned}
$$

Notice the absence of "self-interactions" of the fermionic roots $w_{l}$. This allows us to eliminate $^{3}$ them in the present situation with the following argument. Introduce

$$
q(w):=\prod_{j=1}^{M-1}\left(w-v_{j}+\frac{i}{2}\right)-\prod_{j=1}^{M-1}\left(w-v_{j}-\frac{i}{2}\right) .
$$

$q(w)$ is clearly a polynomial of degree $M-2$ in $w$. It therefore has $M-2$ algebraic roots, which, in light of the last of the above Bethe equations, are precisely the fermionic roots $w_{l}$. We may therefore also write

$$
q(w)=i(M-1) \prod_{l=1}^{M-2}\left(w-w_{l}\right) .
$$

This allows us to deduce that for all $1 \leq j \leq(M-1)$

$$
\prod_{l=1}^{M-2} \frac{v_{j}-w_{l}-\frac{i}{2}}{v_{j}-w_{l}+\frac{i}{2}}=\frac{q\left(v_{j}-\frac{i}{2}\right)}{q\left(v_{j}+\frac{i}{2}\right)}=\prod_{\substack{j^{\prime}=1 \\ j^{\prime} \neq j}}^{M-1} \frac{v_{j}-v_{j^{\prime}}-i}{v_{j}-v_{j^{\prime}}+i} .
$$

This however means that the second set of Bethe equations in (2.36) simplifies significantly:

$$
1=\prod_{k=1}^{M} \frac{v_{j}-u_{k}-\frac{i}{2}}{v_{j}-u_{k}+\frac{i}{2}}
$$

\footnotetext{
${ }^{3}$ The following arguments were also independently discovered by K. Zarembo (private communication) [4].
} 
What has happened is that the fermionic roots $w_{l}$ completely screen the "selfinteraction" terms of the bosonic roots $v_{j}$, such that these become fermionic. Clearly this is a general phenomenon (for this particular excitation pattern) and could be described pictorially as:

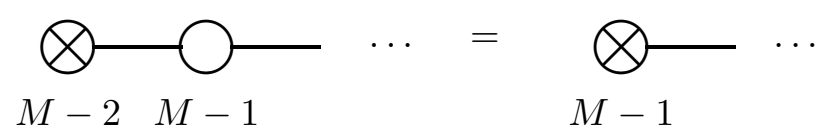

In the case of $\mathfrak{s u}(1 \mid 1)$ we can now iterate the procedure, see diagram (2.35), and thus derive indeed the "free" Bethe equations (2.24) (as first noticed in [30]) written in the Bethe root plane:

$$
\left(\frac{u_{k}+\frac{i}{2}}{u_{k}-\frac{i}{2}}\right)^{L}=1 \quad k=1, \ldots, M .
$$

Notice that the locations $u_{k}$ of the Bethe roots are identical in the super spin chain and the fermionic spin chain formulation, which is far from obvious when superficially comparing (2.36) and (2.42). In fact, this is in line with the intuition that the central roots $u_{k}$ roots encode, via $u_{k}=\frac{1}{2} \cot \frac{p_{k}}{2}$, the momenta of the physical excitations. These clearly should not depend on the description.

Turning to the the $\mathfrak{s l}(2)$ derivative sector of section 2.3, we extract from [3] that the excitation pattern of the states (2.26) is $M_{4}=M, M_{3}=M_{5}=M-1$, $M_{2}=M_{6}=M-2$ and $M_{1}=M_{7}=0$. But this means that we can again apply the above reduction scheme as symbolized in (2.41), this time on both sides of the super Dynkin diagram (2.34). We thus immediately verify that

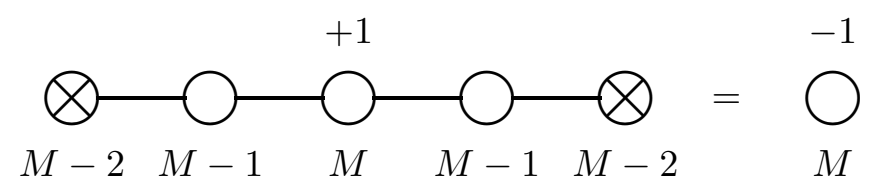

This means that now the auxiliary roots "anti-screen" the interactions of the roots on the central node, and we have indeed derived the Bethe equations (2.33) directly from the super chain. This result opens the interesting possibility to study the distribution of the auxiliary roots in the thermodynamic limit, $c f$ [24], as it is now fairly clear how to find their locations given the above discussion.

\section{Three-Loop S-Matrix for the $\mathcal{N}=4$ Fermionic Sector}

Now we would like to illustrate that the principle of non-diffractive scattering is also very powerful beyond the one-loop level. Let us apply it to the case of $\mathfrak{s u}(1 \mid 1)$ where the higher loop Bethe ansatz is not yet known. However, we do know the 
Hamiltonian up to three loops in algorithmic form from the work of [14]. Rewriting it in spin chain form, we find the following two-loop correction ${ }^{4}$ to the XY model Hamiltonian (2.18) of section 2.2:

$$
\begin{aligned}
H_{2}=\sum_{x=1}^{L} & \left(2\left(\sigma_{x}^{3}-1\right)-\frac{1}{4}\left(\sigma_{x}^{3} \sigma_{x+1}^{3}-1\right)+\frac{9}{8}\left(\sigma_{x}^{1} \sigma_{x+1}^{1}+\sigma_{x}^{2} \sigma_{x+1}^{2}\right)-\right. \\
& -\frac{1}{16}\left(\sigma_{x}^{1} \sigma_{x+1}^{1}+\sigma_{x}^{2} \sigma_{x+1}^{2}\right) \sigma_{x+2}^{3}-\frac{1}{16} \sigma_{x}^{3}\left(\sigma_{x+1}^{1} \sigma_{x+2}^{1}+\sigma_{x+1}^{2} \sigma_{x+2}^{2}\right)- \\
& \left.-\frac{1}{8} \sigma_{x}^{1}\left(1+\sigma_{x+1}^{3}\right) \sigma_{x+2}^{1}-\frac{1}{8} \sigma_{x}^{2}\left(1+\sigma_{x+1}^{3}\right) \sigma_{x+2}^{2}\right) .
\end{aligned}
$$

While it certainly has not yet been rigorously proved that it corresponds to an integrable deformation of the one-loop Hamiltonian, spectral studies for small operators in [14 are consistent with integrability in that certain tell-tale degeneracies (the socalled planar pairs argued to be a hallmark of integrability in [2]) indeed reappear. Further, compelling, evidence will come from the success of the computations below, as they establish that the spectrum may indeed be obtained from the principle of factorized scattering.

If one naively extends the approach of section 2.2 to the higher loop case one quickly finds that the two-body Bethe ansatz (2.7) for the position space wave function $\Psi\left(x_{1}, x_{2}\right)$, defined in (2.19), becomes inconsistent. However, we would still expect that the general form of the Bethe ansatz, namely a superposition of an inand outgoing plane wave, is appropriate when the particles are farther apart than the range of the interaction, which is, in our case, the considered order of perturbation theory:

$$
\Psi\left(x_{1}, x_{2}\right) \sim e^{i p_{1} x_{1}+i p_{2} x_{2}}+S\left(p_{2}, p_{1}\right) e^{i p_{2} x_{1}+i p_{1} x_{2}} \quad \text { if } \quad x_{1} \ll x_{2} .
$$

This is Sutherland's "asymptotic" Bethe ansatz [45]. It was used by Inozemtsev to find the Bethe ansatz for the hyperbolic version of his spin chain [46], and adapted in [15] to diagonalize the three loop dilatation operator in the bosonic $\mathfrak{s u}(2)$ sector.

If the ansatz (3.2) is true, the Schrödinger equation will be satisfied in the asymptotic region $x_{1} \ll x_{2}$ with the energy value given by the sum over the energies of the individual partons. One checks that the three-loop dispersion law of the $\mathfrak{s u}(1 \mid 1)$ Hamiltonian is the same as in the case of the $\mathfrak{s u}(2)$ chain [15], and we thus have

$$
E=\sum_{k=1}^{M}\left(4 \sin ^{2}\left(\frac{p_{k}}{2}\right)-8 g^{2} \sin ^{4}\left(\frac{p_{k}}{2}\right)+32 g^{4} \sin ^{6}\left(\frac{p_{k}}{2}\right)+\mathcal{O}\left(g^{6}\right)\right),
$$

\footnotetext{
${ }^{4}$ The three-loop piece $\mathrm{H}_{4}$ of [14 has been recorded (in momentum space form) in 30; we are refraining from converting it into spin chain form as it is lengthy and little instructive. We found it simplest to use Beisert's original code, and thank him for providing it.
} 
where for the time being $M=2$. Furthermore, the S-matrix $S\left(p_{1}, p_{2}\right)$ may be extracted from the asymptotics (3.2), and we expect it to still be given by a pure phase

$$
S\left(p_{1}, p_{2}\right)=-e^{i \theta\left(p_{1}, p_{2}\right)}
$$

How can we find this phase factor? We will need to adapt the method of the asymptotic Bethe ansatz to the present situation, since, unlike in Inozemtsev's case, here we currently know the Hamiltonian to three loops only. This may be done by modifying the "fine structure" of the wave function close to the collision point, a technique one might term PABA (Perturbative Asymptotic Bethe Ansatz). We make the ansatz $\left(x_{1}<x_{2}\right)$, accurate to $\mathcal{O}\left(g^{4}\right)$ :

$$
\begin{aligned}
\Psi\left(x_{1}, x_{2}\right)= & \left(1+B_{2}\left(p_{1}, p_{2}\right) g^{2\left(x_{2}-x_{1}\right)}+B_{4}\left(p_{1}, p_{2}\right) g^{2+2\left(x_{2}-x_{1}\right)}\right) e^{i p_{1} x_{1}+i p_{2} x_{2}}- \\
& -\left(1+C_{2}\left(p_{1}, p_{2}\right) g^{2\left(x_{2}-x_{1}\right)}+C_{4}\left(p_{1}, p_{2}\right) g^{2+2\left(x_{2}-x_{1}\right)}\right) e^{i p_{2} x_{1}+i p_{1} x_{2}-i \theta\left(p_{1}, p_{2}\right)} \\
& +\mathcal{O}\left(g^{6}\right)
\end{aligned}
$$

Note that this form of the wave function is clearly consistent with the asymptotic ansatz (3.2). The intuition behind (3.5) is that the number of powers of the coupling $g^{2}$ indicates the interaction range on the lattice. It should be fairly clear how to extend the ansatz to even higher loop order.

Acting with the three-loop Hamiltonian ${ }^{5}$ on this wavefunction leads to difference equations similar to (but obviously more involved than) (2.20),(2.21). Substituting the perturbative asymptotic Bethe ansatz (3.5) into these equations, one finds, after somewhat tedious but straightforward computations, that the Schrödinger equation for the nearest $\left(x_{2}=x_{1}+1\right)$, next-nearest $\left(x_{2}=x_{1}+2\right)$ and next-to-next-nearest situation $\left(x_{2}=x_{1}+3\right)$ may be satisfied if we carefully fine-tune the amplitude correction factors $B_{2}\left(p_{1}, p_{2}\right), C_{2}\left(p_{1}, p_{2}\right)$ and $B_{4}\left(p_{1}, p_{2}\right), C_{4}\left(p_{1}, p_{2}\right)$. At two loops one finds the linear condition

$$
C_{2}\left(p_{2}, p_{1}\right)=\frac{1}{4}-\frac{1}{4} e^{2 i p_{2}-2 i p_{1}}+e^{2 i p_{2}-2 i p_{1}} B_{2}\left(p_{1}, p_{2}\right)
$$

At three loops, the functions $B_{2}\left(p_{1}, p_{2}\right)$ and $C_{2}\left(p_{1}, p_{2}\right)$ become fully determined, but their detailed form is non-universal as it depends on various gauge parameters. Furthermore, one now has a new linear constraint, similar to, but significantly more complicated than (3.6), which relates $B_{4}\left(p_{1}, p_{2}\right)$ to $C_{4}\left(p_{1}, p_{2}\right)$. We will not display it as it is not very instructive, and also gauge dependent. The two-body phase shift is

\footnotetext{
${ }^{5}$ Note that that the higher loop Hamiltonians are not uniquely determined and allow for a number of "gauge parameters", $c f$ [14]. Some of these will affect the specific form of the Hamiltonian, as well as the wavefunction correction factors, but not the S-matrix (and therefore the spectrum).
} 
then, to three-loop order, derived to be:

$$
\begin{aligned}
\theta\left(p_{1}, p_{2}\right)= & 4 g^{2} \sin \left(\frac{p_{1}}{2}\right) \sin \left(\frac{p_{1}-p_{2}}{2}\right) \sin \left(\frac{p_{2}}{2}\right)+ \\
& +g^{4} \sin \left(\frac{p_{1}}{2}\right)\left(\sin \left(\frac{p_{1}-3 p_{2}}{2}\right)-7 \sin \left(\frac{p_{1}-p_{2}}{2}\right)+\right. \\
& \left.\quad+\sin \left(\frac{3 p_{1}-3 p_{2}}{2}\right)+\sin \left(\frac{3 p_{1}-p_{2}}{2}\right)\right) \sin \left(\frac{p_{2}}{2}\right)+ \\
& +\mathcal{O}\left(g^{6}\right) .
\end{aligned}
$$

There are many equivalent forms to write this phase shift; a further, interesting one is

$$
\begin{aligned}
\theta\left(p_{1}, p_{2}\right)= & \left(2 g^{2} \sin ^{2}\left(\frac{p_{1}}{2}\right) \sin p_{2}-2 g^{4} \sin ^{4}\left(\frac{p_{1}}{2}\right) \sin \left(2 p_{2}\right)+\right. \\
& \left.+8 g^{4} \sin p_{1} \sin ^{2}\left(\frac{p_{1}}{2}\right) \sin ^{2}\left(\frac{p_{2}}{2}\right)+\mathcal{O}\left(g^{6}\right)\right)- \\
& -\left(p_{1} \leftrightarrow p_{2}\right) .
\end{aligned}
$$

The final steps are again identical to the one loop case. Upon imposing periodic boundary conditions, taking into account Fermi statistics, the Bethe equations are expected to be

$$
e^{i p_{k} L}=\prod_{\substack{j=1 \\ j \neq k}}^{M} e^{i \theta\left(p_{k}, p_{j}\right)}
$$

with $\sum_{k=1}^{M} p_{k}=0$. Now, since we already know their explicit one-loop solution from section 2.2 we can go further and solve our equations (3.9) exactly to three-loop order. Taking a logarithm, we find the fundamental equation $(k=1, \ldots, M)$

$$
p_{k} L=2 \pi n_{k}+\sum_{\substack{j=1 \\ j \neq k}}^{M} \theta\left(p_{k}, p_{j}\right)
$$

which is the higher loop generalization of the free fermion result (2.25). Since we know the scattering phase shift $\theta\left(p_{k}, p_{j}\right)$ exactly to three loops, $c f$ (3.7), we may solve the fundamental equations recursively to $\mathcal{O}\left(g^{4}\right)$ and thus find the loop corrections to the free fermion momenta (2.25). Finally we substitute the obtained momenta $p_{k}$ into the expression (3.3) for the energy, keeping all terms to precision $\mathcal{O}\left(g^{4}\right)$. This procedure then leads to the following explicit result for the, respectively, one-, two- 
and three-loop anomalous dimensions, where $E_{0,2,4}=E_{0,2,4}\left(L, M,\left\{n_{i}\right\}\right)$ :

$$
\begin{aligned}
E_{0}= & 4 \sum_{k=1}^{M} \sin ^{2}\left(\frac{\pi n_{k}}{L}\right) \\
E_{2}=- & 8 \sum_{k=1}^{M} \sin ^{4}\left(\frac{\pi n_{k}}{L}\right)+ \\
& +\frac{16}{L} \sum_{k, j=1}^{M} \cos \left(\frac{\pi n_{k}}{L}\right) \sin ^{2}\left(\frac{\pi n_{k}}{L}\right) \sin \left(\frac{\pi n_{j}}{L}\right) \sin \left(\frac{\pi\left(n_{k}-n_{j}\right)}{L}\right) \\
E_{4}= & 32 \sum_{k=1}^{M} \sin ^{6}\left(\frac{\pi n_{k}}{L}\right)+ \\
& -\frac{16}{L} \sum_{k, j=1}^{M} \cos \left(\frac{\pi n_{k}}{L}\right) \sin ^{2}\left(\frac{\pi n_{k}}{L}\right) \sin \left(\frac{\pi n_{j}}{L}\right) \sin \left(\frac{\pi\left(n_{k}-n_{j}\right)}{L}\right) \times \\
& +\frac{16}{L^{2}} \sum_{k, j, m=1}^{M} \cos \left(\frac{\pi n_{k}}{L}\right) \sin \left(\frac{\pi n_{k}}{L}\right) \sin \left(\frac{\pi n_{j}}{L}\right) \sin \left(\frac{\pi n_{m}}{L}\right) \times \\
& \left.+\frac{2}{L^{2}} \sum_{k, j, m=1}^{M} \sin \left(\frac{\pi n_{k}}{L}\right) \sin \left(\frac{\pi n_{m}}{L}\right) \sin \left(\frac{\pi\left(n_{k}-n_{m}\right)}{L}\right) \times \sin \left(\frac{\pi n_{j}}{L}\right)+\sin ^{2}\left(\frac{\pi\left(n_{k}-n_{j}\right)}{L}\right)\right)+ \\
& \left.\times \sin \left(\frac{\pi\left(n_{j}-n_{m}\right)}{L}\right)\left(\frac{2 \pi n_{j}}{L}\right)-\cos \left(\frac{2 \pi\left(n_{k}-n_{j}\right)}{L}\right)\right)
\end{aligned}
$$

where the relation to the gauge theory scaling dimensions of the operators $(2.16)$ is given by

$$
\Delta=L+\frac{1}{2} M+g^{2} E_{0}+g^{4} E_{2}+g^{6} E_{4}+\mathcal{O}\left(g^{8}\right), \quad \text { with } \quad g^{2}=\frac{g_{\mathrm{YM}}^{2} N}{8 \pi^{2}} .
$$

We should stress that these formulas give the explicit, complete three-loop spectrum of planar anomalous dimensions of all $\mathcal{N}=4$ operators of the form $\operatorname{Tr} Z^{L-M} \psi^{M}$. The fact that it is possible to find such a result is one of the amazing consequences of the higher-loop integrability of the $\mathcal{N}=4$ gauge theory, as first conjectured in [2]. They may also be considered as an all-impurity generalization of the $M=2$ formulas first presented in [2] to arbitrary $M$. (Recall that the spectrum of "two-impurity" states agrees in all sectors [33). Indeed, one checks that the expressions in (3.11) 
reduce, after putting $L=J+1$ and $n_{1}=-n_{2}:=n$, to eqs.(5.17) and (8.10) of [2]. One may also successfully compare to the spectrum of the first few lowest states as obtained by direct diagonalization of the three-loop Hamiltonian for small lengths $L$. In particular, Beisert worked out the full spectrum of highest weight states of the sector $\mathfrak{s u}(2 \mid 3)$ up to dimension $\Delta_{0}=8.5$ [14] (see also [19], p.143). While none of the $\mathfrak{s u}(1 \mid 1)$ operators are primaries in $\mathfrak{s u}(2 \mid 3)$, it is straightforward to find the weight of the corresponding primary operator, and compare its anomalous dimension with (3.11). We present the results of this comparison in Table 1. The agreement is perfect.

One may also check that the formulas (3.11) explain the numerical results obtained in [30]. Finally, it is straightforward to extract the leading $1 / J$ correction to the BMN limit, for an arbitrary number of "impurities", from (3.11). The result agrees with near-BMN string theory at two loops, and disagrees at three, as was found (numerically) in [30].

\section{S-Matrices for Quantum Strings at Large Tension}

Let us now turn to the string side and discuss the, admittedly circumstantial, evidence that the quantum sigma model might also be described by elementary excitations living on a circle of length ${ }^{6} L$, and whose scattering is diffractionless. That is, we will try to interpret all available information on the string spectrum in the basic two-component sectors $\mathfrak{s u}(2), \mathfrak{s u}(1 \mid 1)$ and $\mathfrak{s l}(2)$ in the light of a (conjectured) factorized S-matrix. We then expect that the underlying equation describing this spectrum should be of the type of a fundamental equation

$$
p_{k} L=2 \pi n_{k}+\sum_{\substack{j=1 \\ j \neq k}}^{M} \theta\left(p_{k}, p_{j}\right),
$$

with $\sum p_{k}=0$, obtained by taking a logarithm on both sides of the Bethe equation. Here the scattering phase shift $\theta\left(p_{k}, p_{j}\right)$ is related to the S-matrix as $\theta\left(p_{k}, p_{j}\right)=$ $-i \log \left( \pm S\left(p_{k}, p_{j}\right)\right)$ (the upper sign is for bosons and the lower for fermions), $p_{k}$ are the momenta of individual excitations, and the $n_{k}$ are quantum numbers. The total momentum $P=Q_{1}$, the total energy $E=Q_{2}$, as well as all other higher charges $Q_{r}$ are then expected to be given as linear sums over local dispersion laws $q_{r}\left(p_{k}\right)$ :

$$
Q_{r}=\sum_{k=1}^{M} q_{r}\left(p_{k}\right)
$$

\footnotetext{
${ }^{6}$ One of the deep questions relates to the possible meaning of this "length" in the string sigma model. This is clearly related to the question of the nature of the elementary excitations on the string side.
} 
Finite Length Dispersion Laws The first charge $q_{1}\left(p_{k}\right)$ is the momentum $p_{k}$ :

$$
q_{1}\left(p_{k}\right)=p_{k}
$$

In [16] we proposed for the second charge $q_{2}\left(p_{k}\right)$, i.e. for the energy per excitation, the following long-range dispersion law

$$
q_{2}\left(p_{k}\right)=\frac{1}{g^{2}}\left(\sqrt{1+8 g^{2} \sin ^{2}\left(\frac{p_{k}}{2}\right)}-1\right),
$$

with

$$
g^{2}=\frac{\lambda}{8 \pi^{2}}=\frac{g_{\mathrm{YM}}^{2} N}{8 \pi^{2}}
$$

where $\sqrt{\lambda}$ is the string tension. This is simply a lattice version of the famous BMN energy formula [26]; in fact, one may show that this is the only possible lattice discretization of the BMN expression [2, 34].

Furthermore, a study of the dispersion laws for the higher charges in the $\mathfrak{s u}(2)$ sector, up to five loops, suggests [16] that these are likely given by the expressions

$$
q_{r}\left(p_{k}\right)=\frac{2 \sin \left(\frac{r-1}{2} p_{k}\right)}{r-1}\left(\frac{\sqrt{1+8 g^{2} \sin ^{2}\left(\frac{p_{k}}{2}\right)}-1}{2 g^{2} \sin \left(\frac{p_{k}}{2}\right)}\right)^{r-1} .
$$

We expect (4.6) to hold for the elementary excitations in all sectors.

BMN Limit In this limit [26] one takes $L \rightarrow \infty$ while keeping $M=2,3, \ldots$ small. This is a dilute gas approximation, where, except for level matching, i.e. momentum conservation, the excitations, in both gauge and string theory, do not feel each others presence. Put differently [1], we have

$$
\theta\left(p_{k}, p_{j}\right) \simeq 0
$$

in (4.1), i.e. there is no scattering at all! The fundamental equation (4.1) then simply leads to the quantization law of free non-interactive particles on a circle: $p_{k} L=2 \pi n_{k}$. Substituting this solution of the "Bethe equations" into the dispersion law (4.4) one then immediately recovers, via $\Delta=1+g^{2} Q_{2}$ and using $L \simeq J$, the BMN formula

$$
\Delta=J+\sum_{k=1}^{M} \sqrt{1+\lambda^{\prime} n_{k}^{2}}+\frac{\delta \Delta}{J}+\mathcal{O}\left(\frac{1}{J^{2}}\right) \quad \text { with } \quad \lambda^{\prime}=\frac{g_{\mathrm{YM}}^{2} N}{J^{2}} .
$$

where we have also already introduced the notation $\delta \Delta$ for the leading order $\mathcal{O}(1 / J)$ correction to the energy, see below.

As was first suggested in [16, 10], this simple picture leads to an interesting interpretation of the discrepancies between gauge and string theory which show up in 
the near-BMN limit [28] and the Frolov-Tseytlin limit [15], but, strikingly, apparently not in the strict BMN limit: The discrepancy might be due to a change in the Smatrix (and thus the phase shift $\theta\left(p_{k}, p_{j}\right)$ ) as we go from weak to strong coupling ${ }^{7}$. In this picture, the local dispersion laws should hold for arbitrary values of the coupling constant. Let us next discuss these refined limits, where the interactive but integrable nature of the CFT/AdS system begins to emerge.

Near-BMN Limit Here one is interested in the first $\mathcal{O}(1 / J)$ correction to the BMN expression (4.8). One then expects corrections ${ }^{8}$ to the free particle motion:

$$
p_{k}=\frac{2 \pi}{L} n_{k}+\frac{\delta p_{k}}{L^{2}}+\mathcal{O}\left(\frac{1}{L^{3}}\right) .
$$

What happens is that, due to finite volume effects, the dilute gas approximation breaks down and leading order scattering phase shifts have to be taken into account. If the S-matrix is known, the leading momentum shifts are immediately found from the fundamental equation (4.1):

$$
\delta p_{k}=L \sum_{\substack{j=1 \\ j \neq k}}^{M} \theta\left(\frac{2 \pi}{L} n_{k}, \frac{2 \pi}{L} n_{j}\right)
$$

where it is understood that we only keep terms to leading order in $1 / L$ in the expression $\theta\left(\frac{2 \pi}{L} n_{k}, \frac{2 \pi}{L} n_{j}\right)$. Some care has to be taken as the difference between the length $L$ of the system and the R-charge $J$ begins to matter at this order. For the three basic two-component sectors one has $L=J+\nu M$, where, $\nu=1, \frac{1}{2}, 0$ for, respectively $\mathfrak{s u}(2), \mathfrak{s u}(1 \mid 1)$ and $\mathfrak{s l}(2)$. Expanding the dispersion law (4.4) to this order, we find the following near-BMN energy shift

$$
\delta \Delta=\lambda^{\prime} \sum_{k=1}^{M} \frac{n_{k}}{\sqrt{1+\lambda^{\prime} n_{k}^{2}}}\left(\frac{\delta p_{k}}{2 \pi}-\nu M n_{k}\right) .
$$

We may then combine (4.10) and (4.11) into the final formula

$$
\delta \Delta=\lambda^{\prime} \sum_{\substack{k, j=1 \\ j \neq k}}^{M} \frac{n_{k}}{\sqrt{1+\lambda^{\prime} n_{k}^{2}}}\left(\frac{L}{2 \pi} \theta\left(\frac{2 \pi}{L} n_{k}, \frac{2 \pi}{L} n_{j}\right)+\nu\left(n_{j}-n_{k}\right)\right) .
$$

\footnotetext{
${ }^{7}$ It will also be apparent that both limits probe the phase function and thus the S-matrix to leading order in small values for the individual excitation momenta. These are, unlike in finite length gauge theory, of order $\mathcal{O}(1 / L)$ in both the near-BMN and FT situation. There are indeed indications that the discrepancies might worsen when higher corrections (i.e. $1 / J^{2}$ corrections to BMN and $1 / J$ corrections to FT) are considered [47, 48]. This is entirely consistent with our suspicion that the S-matrix is to be blamed. It presumably changes as one goes from weak to strong coupling.

${ }^{8}$ There are some subtleties, not important for the present discussions, when some of the mode numbers coincide [1, 10]. It was explained in detail in [10] how to deal with them, and the procedure immediately applies to the more general setting discussed here.
} 
Expressions of this structure are indeed found in recent studies of the exact multioscillator quantization of strings in the near-plane wave geometry [29, 31]. Below, cf section 4.2, we will use this connection in reverse, and obtain important information on the string S-matrices from the results of [29, 31].

Frolov-Tseytlin Limit In the thermodynamic limit, around the ferromagnetic vacuum corresponding to BPS states, the fundamental equation (4.1) is expected to turn into an integral equation after the rescaling $p_{k} L \rightarrow p_{k}$ 23, 24, 15, 16]

$$
p(\varphi)=2 \pi n_{\nu}+f_{\mathbf{C}} d \varphi^{\prime} \rho\left(\varphi^{\prime}\right) \theta\left(\varphi, \varphi^{\prime}\right) \quad \text { with } \quad \varphi \in \mathbf{C}_{\nu} .
$$

This limit was originally considered in order to to compare gauge and string theory, following an inspiring proposal by Frolov and Tseytlin [22]. Note that (4.13) is analogous to the equations (4.9), (4.10) of the near-BMN situation in that we expand the phase shift to the same leading order in $\mathcal{O}(1 / L)$. The only difference is that a large number $M=\mathcal{O}(L)$ of excitations have degenerate mode numbers, which requires to work out how their mutual degeneracy is lifted. (This is analogous to the refined near-BMN situation where one also considers coinciding mode numbers.) In (4.13) $\varphi$ is a convenient spectral parameter which leads to a simple form of the momentum $p=p(\varphi)$, all other charges $q_{r}(\varphi):=q_{r}(p(\varphi))$ as well as the phase shift $\theta\left(\varphi, \varphi^{\prime}\right):=\theta\left(p(\varphi), p\left(\varphi^{\prime}\right)\right)$. The discrete "Bethe roots" $\varphi_{k}$ are expected to densely assemble on a union of smooth contours $\mathbf{C}=\mathbf{C}_{1} \cup \mathbf{C}_{2} \ldots$, such that all roots on each component $\mathbf{C}_{\nu}$ carry the same quantum number $n_{\nu}$. Note that this excludes the existence of this particular type of thermodynamic limit in the fermionic sector, as Fermi statistics does not allow coinciding quantum numbers. $\rho(\varphi)$ is the distribution density of the Bethe roots $\varphi$, with support on $\mathbf{C}$ in the complex plane. It is normalized as

$$
\frac{M}{L}=\int_{\mathbf{C}} d \varphi \rho(\varphi),
$$

i.e. it counts the number of excitations in units of the length of the circle (however, cf footnote at the beginning of this chapter). The charges (4.2) are then given in the thermodynamic limit, after rescaling $q_{r} L^{r} \rightarrow q_{r}$ and $Q_{r} L^{r} \rightarrow Q_{r}$, by

$$
Q_{r}=\int_{\mathbf{C}} d \varphi \rho(\varphi) q_{r}(\varphi) .
$$

Equations very similar to (4.13), 4.14), (4.15) are indeed found in recent studies of semiclassical strings moving on the sphere [9], or on AdS [21]. In [10 we used this connection in reverse, and obtained important information on the string S-matrix of the $\mathfrak{s u}(2)$ sector from the results of [9]. The salient points will be briefly reviewed in the next section 4.1. Later we show in section 4.3 that similar results may be obtained for the $\mathfrak{s l}(2)$ sector, where equations resembling Bethe equations have also become available [21]. 


\subsection{The $\mathfrak{s u}(2)$ Bosonic Sector}

In [9] an equation describing the semiclassical spectrum of strings moving with two large angular momenta on the five-sphere was obtained. We would expect the corresponding states to be related to gauge theory operators in the $\mathfrak{s u}(2)$ sector [22]. This equation reads

$$
\frac{x+2 \omega^{2} E x}{x^{2}-\omega^{2}}=2 \pi n_{\nu}+2 f_{\mathcal{C}} d x^{\prime} \frac{\sigma\left(x^{\prime}\right)}{x-x^{\prime}} \quad \text { with } \quad x \in \mathcal{C}_{\nu}
$$

with the energy ${ }^{9} E$ and the momentum $P$ given by

$$
E=\int_{\mathcal{C}} d x \frac{\sigma(x)}{x^{2}} \quad \text { and } \quad P=\int_{\mathcal{C}} d x \frac{\sigma(x)}{x},
$$

where $P$ is quantized by an integer $m: P=2 \pi m$, and the coupling constant $\omega$ appropriate for the thermodynamic limit is

$$
\omega^{2}=\frac{g^{2}}{2 L^{2}}=\frac{g_{\mathrm{YM}}^{2} N}{16 \pi^{2} L^{2}} .
$$

(For comparison, note that $\omega^{2}$ is denoted by $T=\omega^{2}$ in [9, 21].)

The spectral equation (4.16) superficially resembles the fundamental equation (4.13) of the scattering approach, and one might be tempted to identify the spectral parameter $x$ with $\varphi$ in (4.13). However, the fact that an extensive quantity, namely the energy $E$, appears on the left hand side of (4.16) prevents us from interpreting the latter as a parton momentum $p(x)$. A related problem is that the function $\sigma(x)$ is found in [9] to be normalized as

$$
\frac{M}{L}=\int_{\mathcal{C}} d x \sigma(x)\left(1-\frac{\omega^{2}}{x^{2}}\right),
$$

which does not allow for an interpretation of $\sigma(x)$ as an excitation density as in (4.14). However, it was shown in [16] that both problems may be solved "in one go" if we change spectral parameters from $x$ to $\varphi$ according to

$$
\varphi=x+\frac{\omega^{2}}{x}, \quad \text { with } \quad \rho(\varphi):=\sigma(x) .
$$

For one, the quantity $\rho(\varphi)$ in (4.20) is then indeed normalized as in (4.14), and secondly, the spectral equation (4.16) may now be rewritten in the form of a fundamental scattering equation (4.13) with the following dependence of the excitation momenta on the spectral parameter:

$$
p(\varphi)=\frac{1}{\sqrt{\varphi^{2}-4 \omega^{2}}} .
$$

\footnotetext{
${ }^{9}$ In much of the literature on the sigma model, including [10], the total string energy is $1+2 \omega^{2} E$. Here we prefer to continue to use spin chain terminology where the energy $E$ is the eigenvalue of the Hamiltonian, i.e. the anomalous part of the dilatation operator divided by $g^{2}$. We will also continue to use the same symbols in the finite $L$ and the (rescaled) large $L$ cases, as it should always be clear from the context what is meant.
} 
The two-body phase shift is then found to be

$$
\theta_{\mathfrak{s u}(2)}^{\text {string }}\left(\varphi, \varphi^{\prime}\right)=2 \theta_{0}\left(\varphi, \varphi^{\prime}\right)+2 \sum_{r=2}^{\infty} \theta_{r}\left(\varphi, \varphi^{\prime}\right)
$$

where

$$
\theta_{0}\left(\varphi, \varphi^{\prime}\right)=\frac{1}{\varphi-\varphi^{\prime}}
$$

with an infinite number of additional contributions $2 \theta_{r}$ which may be suggestively written as 10

$$
\theta_{r}\left(\varphi, \varphi^{\prime}\right)=\omega^{2 r}\left(q_{r}(\varphi) q_{r+1}\left(\varphi^{\prime}\right)-q_{r+1}(\varphi) q_{r}\left(\varphi^{\prime}\right)\right)
$$

Here the $q_{r}$ are found to be given by the expressions

$$
q_{r}(\varphi)=\frac{1}{\sqrt{\varphi^{2}-4 \omega^{2}}} \frac{1}{\left(\frac{1}{2} \varphi+\frac{1}{2} \sqrt{\varphi^{2}-4 \omega^{2}}\right)^{r-1}} .
$$

It so turns out that these formulas are identical to the thermodynamic limit of our lattice dispersion laws (4.6) when expressed, via (4.21), as functions of the spectral parameter $\varphi$. This is fascinating, as they simply follow from rewriting the semiclassical equations of [9] in a form that unveils the underlying scattering processes!

What is more, recovering the thermodynamic fundamental scattering equation from classical string theory suggests a very natural way to rediscretize it and guess the large tension, small momentum (i.e. large length $L$ ) S-matrix of the quantum sigma model [10]. Here we recall that the proposed [16] long-range S-matrix of the weak-coupling gauge theory in the $\mathfrak{s u}(2)$ sector is

$$
S_{\mathfrak{s u}(2)}^{\text {gauge }}\left(p_{k}, p_{j}\right)=\frac{\varphi\left(p_{k}\right)-\varphi\left(p_{j}\right)+i}{\varphi\left(p_{k}\right)-\varphi\left(p_{j}\right)-i} .
$$

Its logarithm yields, in the thermodynamic limit, the leading contribution $\theta_{0}$, see (4.23), to the string theory two-body scattering kernel. The all-order expression for the lattice phase function $\varphi\left(p_{k}\right)$ is conjectured [16] to be

$$
\varphi\left(p_{k}\right)=\frac{1}{2} \cot \left(\frac{p_{k}}{2}\right) \sqrt{1+8 g^{2} \sin ^{2}\left(\frac{p_{k}}{2}\right)}
$$

and yields in the thermodynamic limit, upon inversion, (4.21). It is then very natural to include the additional scattering terms $\theta_{r}$ of (4.24) as a "dressing factor" to the "bare" S-matrix (4.26) and write down an ansatz for the string S-matrix [10]:

$$
S_{\mathfrak{s u}(2)}^{\text {string }}\left(p_{k}, p_{j}\right) \simeq \frac{\varphi\left(p_{k}\right)-\varphi\left(p_{j}\right)+i}{\varphi\left(p_{k}\right)-\varphi\left(p_{j}\right)-i} \prod_{r=2}^{\infty} e^{2 i \theta_{r}\left(p_{k}, p_{j}\right)},
$$


while replacing (1), via (4.18), the continuum coupling $\omega^{2}$ by the lattice coupling $g^{2}$ and (2) the continuum dispersion laws (4.25) by the lattice laws (4.6):

$$
\theta_{r}\left(p_{k}, p_{j}\right)=\left(\frac{g^{2}}{2}\right)^{r}\left(q_{r}\left(p_{k}\right) q_{r+1}\left(p_{j}\right)-q_{r+1}\left(p_{k}\right) q_{r}\left(p_{j}\right)\right) \text {. }
$$

One now checks, with the help of (4.12), agreement with the near-BMN multiimpurity results of [29, 31], as was first done in [10].

\subsection{The $\mathfrak{s u}(1 \mid 1)$ Fermionic Sector}

In the fermionic sector no "ferromagnetic" thermodynamic limit similar to the one discussed in the last section exists. Due to Fermi statistics the mode numbers of all excitations are distinct. This prevents the Bethe roots from condensing onto smooth cuts in the complex spectral parameter plane, where the mode number has to stay constant along each contour. Luckily there is an alternative way to deduce information on the string S-matrix of this sector. In a beautiful paper Callan, McLoughlin and Swanson [29] studied the spectrum of three elementary string excitations in the near-BMN limit. It so turns out that this is precisely what we need! In section 2 we argued that, given integrability, it suffices to carefully solve the two-body problem in order to deduce the S-matrix. It is however important that the two excitations are "off-shell", i.e. they need to be capable of carrying arbitrary momenta. Now, since the level matching condition in string theory enforces the total momentum conservation of all excitations, the solution of the two-impurity problem 28] does not contain enough information as the absolute values of the two associated momenta are necessarily equal. This is no longer the case if we decompose the three-body scattering into a series of two-body processes. And indeed, the principle of factorized scattering then immediately yields the solution of the M-body problem, as was intuitively and correctly understood in a nice follow-up paper by McLoughlin and Swanson [31]. Rewritten in our present notations, they find ( $c f$ eq.(3.30) in [31]) the following energy shift for $M$ excitations in the $\mathfrak{s u}(1 \mid 1)$ sector:

$$
\delta \Delta=-\frac{\lambda^{\prime}}{4} \sum_{\substack{k, j=1 \\ j \neq k}}^{M}\left(\frac{n_{k}^{2}+n_{j}^{2}+2 \lambda^{\prime} n_{k}^{2} n_{j}^{2}}{\sqrt{1+\lambda^{\prime} n_{k}^{2}} \sqrt{1+\lambda^{\prime} n_{j}^{2}}}-2 n_{k} n_{j}\right) .
$$

This double sum may be rearranged as

$$
\begin{aligned}
\delta \Delta=\lambda^{\prime} \sum_{\substack{k, j=1 \\
j \neq k}}^{M} & \frac{n_{k}}{\sqrt{1+\lambda^{\prime} n_{k}^{2}}} \times \\
& \times\left(\frac{1}{2} n_{j}\left(\sqrt{1+\lambda^{\prime} n_{k}^{2}}-1\right)-\frac{1}{2} n_{k}\left(\sqrt{1+\lambda^{\prime} n_{j}^{2}}-1\right)+\frac{1}{2}\left(n_{j}-n_{k}\right)\right)
\end{aligned}
$$


By comparing to our general formula (4.12) (here $\nu=\frac{1}{2}$ as appropriate for the fermionic sector), we may then read of the phase shift, accurate to leading order in small $p_{k}, p_{j}$, as

$$
-\theta_{1}\left(p_{k}, p_{j}\right) \simeq-\frac{g^{2}}{2}\left(p_{k} q_{2}\left(p_{j}\right)-q_{2}\left(p_{k}\right) p_{j}\right)
$$

where we have used (4.4) (for small $p_{k}$ ) and employed the notation $\theta_{r}\left(p_{k}, p_{j}\right)$, introduced in (4.29), with $r=1$. This then leads to the following simple, approximate S-matrix encoding the near-BMN string physics in the fermi sector:

$$
S_{\mathfrak{s u}(1 \mid 1)}^{\text {string }}\left(p_{k}, p_{j}\right) \simeq-e^{-i \theta_{1}\left(p_{k}, p_{j}\right)}
$$

\subsection{The $\mathfrak{s l}(2)$ Derivative Sector}

In the case of the third basic two-component sector $\mathfrak{s l}(2)$ we have a choice for extracting the S-matrix from known string theory results. We could either proceed as in the last section 4.2, using again (4.12) in conjunction with the multi-impurity near-BMN results of [29, 31]. Alternatively we can apply once more the logic of section 4.1 and test them on the semiclassical string sigma model results of Kazakov and Zarembo which have recently become available [21]. Interestingly, both procedures lead to the same final result, showing once more the close connection between the leading curvature corrections to quantum strings in a plane wave geometry, and semiclassical strings on the curved geometry $A d S_{5} \times S^{5}$. Let us proceed in the second fashion, which will serve as a nice check on the ideas presented in section 4.1.

For semiclassical strings rotating with one large angular momentum on the fivesphere and one large spin on AdS, the bootstrap equation derived in 21] from the monodromy matrix of the classically integrable string sigma model reads

$$
\frac{x-2 \omega^{2} P}{x^{2}-\omega^{2}}=2 \pi n_{\nu}-2 f_{\mathcal{C}} d x^{\prime} \frac{\sigma\left(x^{\prime}\right)}{x-x^{\prime}} \quad \text { with } \quad x \in \mathcal{C}_{\nu},
$$

where the function $\sigma(x)$ is normalized exactly as in the $S^{5}$ case according to formula (4.19) (now $M$ is the spin quantum number on $\mathrm{AdS}_{5}$, whereas previously $M$ was one of the two angular momenta on $\mathrm{S}^{5}$, see also [24]). The equation (4.34) is manifestly very similar to the corresponding equation (4.16) of the $\mathfrak{s u}(2)$ sector. Again an extensive quantity appears on the left side of this equation, preventing its naive interpretation as a fundamental scattering equation: This time it is not the energy $E$ but the total momentum $P$, see (4.17). However, since the normalization conditions (4.19) are identical in the two cases, it is natural to once more apply the same change of spectral parameter (4.20). This converts (4.34) to the form of a fundamental scattering equation, see (4.13)

$$
p(\varphi)=2 \pi n_{\nu}-f_{\mathbf{C}} d \varphi^{\prime} \rho\left(\varphi^{\prime}\right)\left(\frac{2}{\varphi-\varphi^{\prime}}+2 \sum_{r=1}^{\infty} \theta_{r}\left(\varphi, \varphi^{\prime}\right)\right)
$$


where $\varphi \in \mathbf{C}_{\nu}$, the momentum is parametrized as before through (4.21), the density $\rho(\varphi)$ is consistently normalized as in (4.14), and the phase shifts $\theta_{r}\left(\varphi, \varphi^{\prime}\right)$ are given in (4.24). We may then read off the continuum two-body scattering phase shift of the $\mathfrak{s l}(2)$ sector as

$$
\theta_{\mathfrak{s l}(2)}^{\text {string }}\left(\varphi, \varphi^{\prime}\right)=-2 \theta_{0}\left(\varphi, \varphi^{\prime}\right)-2 \sum_{r=1}^{\infty} \theta_{r}\left(\varphi, \varphi^{\prime}\right),
$$

which differs from the one of the $\mathfrak{s u}(2)$ sector (4.22) by (1) an overall minus sign and (2) the fact that the sum starts at $r=1$ instead of $r=2$.

As a consistency check we may verify that the near-BMN physics is properly reproduced. Replacing the shift (4.36) by its discrete, small $p_{k}$ (i.e. large $L$ ) version in the, by now, familiar fashion, we find

$$
\theta\left(p_{k}, p_{j}\right) \simeq-\frac{2}{\varphi\left(p_{k}\right)-\varphi\left(p_{j}\right)}-2 \sum_{r=1}^{\infty} \theta_{r}\left(p_{k}, p_{j}\right),
$$

where the lattice expressions (4.27) and (4.29) should be used (replacing momenta by their leading $\mathcal{O}(1 / L)$ approximations). If we now insert (4.37) into the formula (4.12) for the energy shift (with $\nu=0$ for the $\mathfrak{s l}(2)$ sector) we reproduce, after a short calculation, indeed the $\mathfrak{s l}(2)$ multi-impurity result of [29, 31]

$$
\delta \Delta=\frac{\lambda^{\prime}}{2} \sum_{\substack{k, j=1 \\ j \neq k}}^{M}\left(\frac{n_{k} n_{j}-\lambda^{\prime} n_{k}^{2} n_{j}^{2}}{\sqrt{1+\lambda^{\prime} n_{k}^{2}} \sqrt{1+\lambda^{\prime} n_{j}^{2}}}+n_{k} n_{j}\right) .
$$

cf equation (3.24) in [31]. The case of coinciding mode numbers is dealt with exactly as in [10], and immediately reproduces equation (3.23) of [31.

Finally we may write the (large tension, small momentum) S-matrix for the $\mathfrak{s l}(2)$ sector suggested by the phase shift (4.37):

$$
S_{\mathfrak{s l}(2)}^{\text {string }}\left(p_{k}, p_{j}\right) \simeq \frac{\varphi\left(p_{k}\right)-\varphi\left(p_{j}\right)-i}{\varphi\left(p_{k}\right)-\varphi\left(p_{j}\right)+i} \prod_{r=1}^{\infty} e^{-2 i \theta_{r}\left(p_{k}, p_{j}\right)}
$$

Thus the approximate string S-matrix in the $\mathfrak{s l}(2)$ sector differs from the one of the $\mathfrak{s u}(2)$ sector (4.28) of 10 by (1) complex conjugating the explicit factors of $i \rightarrow-i$ and (2) by one additional factor with $r=1$.

\section{Three-Loop S-Matrix for the $\mathcal{N}=4$ Derivative Sector}

Observe the following striking relationship between the conjectured large string tension, small momentum S-matrices (4.28),(4.33),(4.39) of the three basic twocomponent sectors:

$$
S_{\mathfrak{s l}(2)}=S_{\mathfrak{s u}(1 \mid 1)} S_{\mathfrak{s u}(2)}^{-1} S_{\mathfrak{s u}(1 \mid 1)}
$$


The simplicity of the relation (5.1) suggests a purely group-theoretical explanation. In fact, it appears to result from an "inversion" of the Dynkin diagram (2.34) where we now place the representation -1 onto the central node; i.e. we exchange the AdS and $\mathrm{S}^{5}$ sectors.

Since the relationship (5.1) seems to be based on symmetry alone it is very reasonable to expect that it also holds at weak coupling, i.e. in the gauge theory. We are thus led to the following Bethe ansatz for the weak-coupling $\mathfrak{s l}(2)$ sector at finite $L=J$ and finite $M=S$ (with $k=1, \ldots, M)$ :

$$
e^{i p_{k} L}=\prod_{\substack{j=1 \\ j \neq k}}^{M}\left(\frac{\varphi\left(p_{k}\right)-\varphi\left(p_{j}\right)-i}{\varphi\left(p_{k}\right)-\varphi\left(p_{j}\right)+i} e^{2 i \theta\left(p_{k}, p_{j}\right)}\right)
$$

where $\varphi(p)$ is given in (4.27) and $\theta\left(p_{k}, p_{j}\right)$ is currently known to three-loop precision, cf 3.7$)$ or (3.8).

A first, non-trivial test of this ansatz may be performed immediately. We know that all two-impurity states must agree for arbitrary, finite R-charge $J$ [33]. Let us compare the $M=2$ Bethe ansätze for the $\mathfrak{s u}(2)$ sectors [15, 16] and the $\mathfrak{s u}(1 \mid 1)$ sectors ( $c f$ section 2.2):

$$
\mathfrak{s u}(2): \quad e^{i p(J+2)}=\frac{\varphi(p)+\frac{i}{2}}{\varphi(p)-\frac{i}{2}} \quad \mathfrak{s u}(1 \mid 1): \quad e^{i p(J+1)}=e^{i \theta(p,-p)}
$$

from which we conclude that we must have to all orders in the coupling constant $g$

$$
e^{i \theta(p,-p)}=e^{-i p} \frac{\varphi(p)+\frac{i}{2}}{\varphi(p)-\frac{i}{2}} .
$$

The reason is that we have the same dispersion relation in all sectors, and the momenta $p_{1}:=p, p_{2}:=-p$ for two impurities must therefore agree in different sectors. We easily check that the three-loop phase shift obtained in section 2.2 satisfies (5.4) to $\mathcal{O}\left(g^{4}\right)$. One now immediately verifies that the ansatz (5.2) for the $\mathfrak{s l}(2)$ sector, where the length is $L=J$, results in a Bethe equation for $p$ which is entirely equivalent to 5.3$)$ :

$$
\mathfrak{s l}(2): \quad e^{i p J}=\frac{\varphi(p)-\frac{i}{2}}{\varphi(p)+\frac{i}{2}} e^{2 i \theta(p,-p)} .
$$

Now, naively we would expect the Bethe ansatz (5.2) to be asymptotic only, that is we may a priori not expect that it properly diagonalizes operators which are shorter than the range of interaction. These are precisely the twist-two operators at two and three loops, and the twist three operators at three loops. However, the consistency check just presented actually yields a further, crucial hint. We notice that our $\mathfrak{s l}(2)$ ansatz also works at least up to three loops for the $M=2$ states with $J=2$ and $J=$ 
3, cf (5.5). But these are, respectively, length two and length three operators. This observation leads to the expectation that in fact all $\mathfrak{s l}(2)$ operators are diagonalized by the Bethe ansatz (5.2)! This allows us to test our ansatz since, luckily, the three-loop anomalous dimensions of twist-two operators in the $\mathcal{N}=4$ gauge theory have recently become available. Kotikov, Lipatov, Onishchenko and Velizhanin [35] were able to extract them, under some unproven but astute assumptions, from an impressive, rigorous computation of three-loop anomalous dimensions in QCD by Moch, Vermaseren and Vogt [36].

We have not worked out the explicit three-loop spectrum of twist two operators from (5.2) for arbitrary even AdS spin $M$ (in the $\mathfrak{s l}(2)$ sector $S=M$ has to be even for twist-two operators), but certainly expect that this will reproduce the general formulas derived in [35] ( $c f$ equations $(10),(11),(12)$ of that paper, where the notation $j=M$ is used), which read:

$$
\begin{aligned}
& E_{0}(M)=4 \Sigma_{1} \\
& \begin{aligned}
E_{2}(M)=-4 & \left(\Sigma_{3}+\Sigma_{-3}-2 \Sigma_{-2,1}+2 \Sigma_{1}\left(\Sigma_{2}+\Sigma_{-2}\right)\right) \\
E_{4}(M)=-8( & 2 \Sigma_{-3} \Sigma_{2}-\Sigma_{5}-2 \Sigma_{-2} \Sigma_{3}-3 \Sigma_{-5}+24 \Sigma_{-2,1,1,1} \\
& +6\left(\Sigma_{-4,1}+\Sigma_{-3,2}+\Sigma_{-2,3}\right)-12\left(\Sigma_{-3,1,1}+\Sigma_{-2,1,2}+\Sigma_{-2,2,1}\right) \\
& -\left(\Sigma_{2}+2 \Sigma_{1}^{2}\right)\left(3 \Sigma_{-3}+\Sigma_{3}-2 \Sigma_{-2,1}\right)-\Sigma_{1}\left(8 \Sigma_{-4}+\Sigma_{-2}^{2}\right. \\
& \left.\left.+4 \Sigma_{2} \Sigma_{-2}+2 \Sigma_{2}^{2}+3 \Sigma_{4}-12 \Sigma_{-3,1}-10 \Sigma_{-2,2}+16 \Sigma_{-2,1,1}\right)\right)
\end{aligned}
\end{aligned}
$$

and the harmonic sums $\Sigma_{ \pm a, b, c, \cdots}:=\Sigma_{ \pm a, b, c, \cdots}(M)$ are defined recursively $(a, b, c>0)$

$$
\Sigma_{ \pm a}(M)=\sum_{m=1}^{M} \frac{( \pm 1)^{m}}{m^{a}}, \quad \Sigma_{ \pm a, b, c, \cdots}(M)=\sum_{m=1}^{M} \frac{( \pm 1)^{m}}{m^{a}} \Sigma_{b, c, \cdots}(m) .
$$

We checked explicitly that our ansatz (5.2) reproduces the result predicted in (5.6) in the cases $M=2,4,6,8$; see also Table 2 .

We would however like to stress that the ansatz (5.2) is expected to also properly reproduce the three-loop anomalous dimensions for operators of arbitrary twist and spin. As far as we know no non-trivial twist-three two-loop, let alone three-loop, anomalous dimensions seem to be known in $\mathcal{N}=4$ gauge theory from a field theory computation to date. For example, for the simplest twist-three field not in the twoimpurity supermultiplet, namely $\operatorname{Tr} D^{3} Z^{3}+\ldots$, our ansatz predicts a paired state with three-loop energy

$$
E=\frac{15}{2}-\frac{225}{16} g^{2}+\frac{3195}{64} g^{4}+\mathcal{O}\left(g^{6}\right) .
$$

It would be exciting if this prediction could be checked by a traditional Feynman diagram computation ${ }^{10}$

${ }^{10}$ After the completion of this manuscript we were informed by B. Eden that the two-loop part of the prediction (5.7) may indeed be confirmed by a full-fledged field theory computation [49. 
Finally we may answer a question raised in the paper [21] about whether the discrepancies between string and gauge theory might already appear in the $\mathfrak{s l}(2)$ sector at two instead of at three loops. Comparing the S-matrix entering the r.h.s. of (5.2) ( $c f$ also (6.4)) and the string S-matrix (4.39) by expanding in small momenta $p_{k}, p_{j}$ of order $\mathcal{O}(1 / L)$ one finds,

$$
-i\left(\log S_{\mathfrak{s l}(2)}^{\text {gauge }}\left(p_{k}, p_{j}\right)-\log S_{\mathfrak{s l}(2)}^{\text {string }}\left(p_{k}, p_{j}\right)\right) \simeq \frac{g^{4}}{2} p_{k}^{2}\left(p_{k}-p_{j}\right) p_{j}^{2}+\mathcal{O}\left(g^{6}\right)
$$

This proves, incidentally for both the near-BMN limit as well as the Frolov-Tseytlin limit, two-loop agreement and three-loop disagreement in the $\mathfrak{s l}(2)$ sector, in full analogy with the $\mathfrak{s u}(2)$ and $\mathfrak{s u}(1 \mid 1)$ sectors.

\section{Summary and Musing}

Let us collect once more the long-range, asymptotic Bethe ansätze we proposed for the basic two-component sectors $\mathfrak{s u}(2), \mathfrak{s u}(1 \mid 1)$ and $\mathfrak{s l}(2)$ of $\mathcal{N}=4$ gauge theory. They are all written in the factorized scattering form

$$
e^{i p_{k} L}=\prod_{\substack{j=1 \\ j \neq k}}^{M} \pm S\left(p_{k}, p_{j}\right), \quad k=1, \ldots, M
$$

where the upper sign is for bosons and the lower for fermions. The three S-matrices are

$$
\begin{aligned}
S_{\mathfrak{s u}(2)}^{\text {gauge }}\left(p_{k}, p_{j}\right) & =\frac{\varphi\left(p_{k}\right)-\varphi\left(p_{j}\right)+i}{\varphi\left(p_{k}\right)-\varphi\left(p_{j}\right)-i} \\
S_{\mathfrak{s u}(1 \mid 1)}^{\text {gauge }}\left(p_{k}, p_{j}\right) & =-e^{i \theta\left(p_{k}, p_{j}\right)} \\
S_{\mathfrak{s l}(2)}^{\text {gauge }}\left(p_{k}, p_{j}\right) & =\frac{\varphi\left(p_{k}\right)-\varphi\left(p_{j}\right)-i}{\varphi\left(p_{k}\right)-\varphi\left(p_{j}\right)+i} e^{2 i \theta\left(p_{k}, p_{j}\right)} .
\end{aligned}
$$

The phase function $\varphi\left(p_{k}\right)$ is currently known to five loops, and its all-loop conjecture is (4.27) [16]. The phase $\theta\left(p_{k}, p_{j}\right)$ was worked out in section 3 to three-loop order, $c f$ (3.7), (3.8). It would be fascinating to find its all-loop form.

On the string side, it was found, for $\mathfrak{s u}(2)$ in 10 , and in section 4 for the other two sectors, that the near-BMN and Frolov-Tseytlin physics is, for large $L$ (i.e. momenta of order $\mathcal{O}(1 / L))$ reproduced to all orders in $g^{2}$ by the S-matrices

$$
\begin{aligned}
S_{\mathfrak{s u}(2)}^{\text {string }}\left(p_{k}, p_{j}\right) & \simeq \frac{\varphi\left(p_{k}\right)-\varphi\left(p_{j}\right)+i}{\varphi\left(p_{k}\right)-\varphi\left(p_{j}\right)-i} \prod_{r=2}^{\infty} e^{2 i \theta_{r}\left(p_{k}, p_{j}\right)} \\
S_{\mathfrak{s u}(1 \mid 1)}^{\text {string }}\left(p_{k}, p_{j}\right) & \simeq-e^{-i \theta_{1}\left(p_{k}, p_{j}\right)} \\
S_{\mathfrak{s l}(2)}^{\text {string }}\left(p_{k}, p_{j}\right) & \simeq \frac{\varphi\left(p_{k}\right)-\varphi\left(p_{j}\right)-i}{\varphi\left(p_{k}\right)-\varphi\left(p_{j}\right)+i} \prod_{r=1}^{\infty} e^{-2 i \theta_{r}\left(p_{k}, p_{j}\right)}
\end{aligned}
$$


Finally, it was demonstrated in [50] that to at least five-loop order a spin chain exists whose S-matrix $\tilde{S}_{\mathfrak{s u}(2)}$ is exactly given by the right hand side of (6.5). Interestingly this model is part of the general three-loop $\mathfrak{s u}(2 \mid 3)$ spin chain of [14, which also contains the $\mathfrak{s u}(1 \mid 1)$ subsector. Therefore supersymmetry predicts that also a fermionic spin chain should exist which reproduces the string and not the gauge theory scattering. However, as opposed to (6.5), the S-matrix (6.6) can unfortunately not, as it stands, correspond to the one of a quantum spin chain. The reason is that the shift function $\theta_{r}\left(p_{k}, p_{j}\right)$ is not periodic ${ }^{11}$ in the momenta $p_{k}, p_{j}$ for $r=1$. Excitingly, one may nevertheless find evidence that the same deformation which turns the gauge theory spin chain into the string theory spin chain in the $\mathfrak{s u}(2)$ sector yields for the $\mathfrak{s u}(1 \mid 1)$ sector an S-matrix $\tilde{S}_{\mathfrak{s u}(1 \mid 1)}$ which is, to three loop order, "dressed" by the same multiplicative factor $\exp 2 i \theta_{2}\left(p_{k}, p_{j}\right)$ as in the $\mathfrak{s u}(2)$ case. In fact, we have checked, by including the corresponding deformation parameter (denoted by $c_{4}$ in [50], where $c_{4}$ is the gauge case and $c_{4}=1$ the string case) into the calculations of section 3 that the deformation leads to the following additional phase shift for $\mathfrak{s u}(1 \mid 1)$

$$
\delta \theta\left(p_{1}, p_{2}\right)=-8 c_{4} g^{4} \sin ^{2}\left(\frac{p_{1}}{2}\right)\left(\sin p_{1}-\sin p_{2}\right) \sin ^{2}\left(\frac{p_{2}}{2}\right)
$$

to be added to (3.8). (Note that in the string case $c_{4}=1$ it exactly cancels the last term in (3.8).) This is possible since the deformation parameter appears in the full three-loop $\mathfrak{s u}(2 \mid 3)$ vertex of [14]. It is therefore reasonable to speculate that a spin chain might exists which reproduces the string results for all sectors and, maybe, all of $\mathfrak{p s u}(2,2 \mid 4)$. For the two-component sectors discussed in this paper this spin chain might have an S-matrix which is related to the weak-coupling, asymptotic gauge theory S-matrix by the same dressing factor:

$$
\begin{aligned}
\tilde{S}_{\mathfrak{s u}(2)}^{\text {string }}\left(p_{k}, p_{j}\right) & =S_{\mathfrak{s u}(2)}^{\text {gauge }}\left(p_{k}, p_{j}\right) \hat{S}\left(p_{k}, p_{j}\right), \\
\tilde{S}_{\mathfrak{s u}(1 \mid 1)}^{\text {string }}\left(p_{k}, p_{j}\right) & =S_{\mathfrak{s u}(1 \mid 1)}^{\text {gauge }}\left(p_{k}, p_{j}\right) \hat{S}\left(p_{k}, p_{j}\right), \\
\tilde{S}_{\mathfrak{s l}(2)}^{\text {string }}\left(p_{k}, p_{j}\right) & =S_{\mathfrak{s l}(2)}^{\text {gauge }}\left(p_{k}, p_{j}\right) \hat{S}\left(p_{k}, p_{j}\right) .
\end{aligned}
$$

where the dressing factor would be approximately given by the product

$$
\hat{S}\left(p_{k}, p_{j}\right) \simeq \prod_{r=2}^{\infty} e^{2 i \theta_{r}\left(p_{k}, p_{j}\right)}
$$

Hopefully such a dressing factor will appear in the gauge theory when we go from weak to strong coupling, e.g. through the "wrapping effects" discussed in 16]. Or do we have to replace the AdS/CFT "correspondence" by an AdS/CFT "similarity"?

\footnotetext{
${ }^{11}$ It should be clear from the perturbative asymptotic Bethe ansatz introduced in this paper that the latter will always yield a periodic S-matrix. The same caveat therefore applies to the $\mathfrak{s l}(2)$ string S-matrix (6.7).
} 
It would be exceedingly important to test these ideas in refined situations such as $1 / J^{2}$ corrections to the near-BMN limit, and $1 / L$ corrections to spinning strings. For first steps in these directions see, respectively, [51 and 447, 48. A dressing factor as in (6.9) should then also explain the famous $\lambda^{\frac{1}{4}}$ strong coupling behavior. First evidence that this might happen was found in [10]. It would also be very interesting to reproduce other semiclassical limits, such as the large spin limit of [52], from the S-matrices.

\section{Acknowledgments}

I thank the Kavli Institute for Theoretical Physics for hospitality, and the organizers of the program $Q C D$ and String Theory for an inspiring workshop. I also thank Ofer Aharony, Gleb Arutyunov, David Berenstein, Zvi Bern, Vladimir Braun, Virginia Dippel, Burkhard Eden, Alexander Gorsky, David Gross, Romuald Janik, Martin Kruczenski, Sven-Olaf Moch, Jan Plefka, Joe Polchinski, Marcus Spradlin, Matt Strassler, Stefan Theisen, Arkady Tseytlin, Anastasia Volovich, Arkady Vainshtein, Kostya Zarembo and, especially, Niklas Beisert for helpful comments, heated discussions, interest, and useful correspondence. Thanks to Gleb Arutyunov, Joe Minahan and Kostya Zarembo for suggestions on the manuscript. This research was supported in part by the National Science Foundation under Grant No. PHY99-07949.

\section{References}

[1] J. A. Minahan and K. Zarembo, "The Bethe-ansatz for $N=4$ super Yang-Mills," JHEP 0303 (2003) 013, hep-th/0212208.

[2] N. Beisert, C. Kristjansen and M. Staudacher, "The dilatation operator of $N=4$ super Yang-Mills theory," Nucl. Phys. B 664 (2003) 131, hep-th/0303060.

[3] N. Beisert and M. Staudacher, "The $N=4$ SYM integrable super spin chain," Nucl. Phys. B 670 (2003) 439, hep-th/0307042.

[4] L. N. Lipatov, "High-energy asymptotics of multicolor QCD and exactly solvable lattice models,” JETP Lett. 59 (1994) 596 [Pisma Zh. Eksp. Teor. Fiz. 59 (1994) 571], hep-th/9311037.

[5] A. V. Belitsky, V. M. Braun, A. S. Gorsky and G. P. Korchemsky, "Integrability in QCD and beyond," Int. J. Mod. Phys. A 19, 4715 (2004), hep-th/0407232.

[6] I. Bena, J. Polchinski and R. Roiban, "Hidden symmetries of the $A d S_{5} \times S^{5}$ superstring," Phys. Rev. D 69 (2004) 046002, hep-th/0305116.

[7] G. Arutyunov, S. Frolov, J. Russo and A. A. Tseytlin, "Spinning strings in $A d S_{5} \times S^{5}$ and integrable systems," Nucl. Phys. B 671, 3 (2003), hep-th/0307191; 
G. Arutyunov, J. Russo and A. A. Tseytlin, "Spinning strings in $A d S_{5} \times S^{5}: N e w$ integrable system relations," Phys. Rev. D 69 (2004) 086009, hep-th/0311004.

[8] G. Arutyunov and M. Staudacher, "Matching higher conserved charges for strings and spins," JHEP 0403 (2004) 004, hep-th/0310182; see also: "Two-loop commuting charges and the string / gauge duality," hep-th/0403077.

[9] V. A. Kazakov, A. Marshakov, J. A. Minahan and K. Zarembo, "Classical / quantum integrability in AdS/CFT," JHEP 0405 (2004) 024 hep-th/0402207.

[10] G. Arutyunov, S. Frolov and M. Staudacher, "Bethe ansatz for quantum strings," JHEP 0410 (2004) 016, hep-th/0406256.

[11] G. Arutyunov and S. Frolov, "Integrable Hamiltonian for classical strings on $A d S_{5} \times S^{5}, "$ hep-th/0411089.

[12] Z. Bern, L. J. Dixon and D. A. Kosower, " $N=4$ super-Yang-Mills theory, $Q C D$ and collider physics," hep-th/0410021.

[13] N. Beisert, "The complete one-loop dilatation operator of $N=4$ super Yang-Mills theory," Nucl. Phys. B 676 (2004) 3, hep-th/0307015.

[14] N. Beisert, “The su(2|3) dynamic spin chain," Nucl. Phys. B 682 (2004) 487, hep-th/0310252.

[15] D. Serban and M. Staudacher, "Planar $N=4$ gauge theory and the Inozemtsev long range spin chain," JHEP 0406 (2004) 001, hep-th/0401057.

[16] N. Beisert, V. Dippel and M. Staudacher, "A novel long range spin chain and planar $N=4$ super Yang-Mills," JHEP 0407 (2004) 075, hep-th/0405001.

[17] T. Klose and J. Plefka, "On the integrability of large N plane-wave matrix theory," Nucl. Phys. B 679, 127 (2004), hep-th/0310232.

[18] B. Eden, C. Jarczak and E. Sokatchev, "A three-loop test of the dilatation operator in $N=4 S Y M$," hep-th/0409009.

[19] N. Beisert, "The dilatation operator of $N=4$ super Yang-Mills theory and integrability," Phys. Rept. 405 (2005) 1, hep-th/0407277.

[20] N. Mann and J. Polchinski, "Finite density states in integrable conformal field theories," hep-th/0408162.

[21] V. A. Kazakov and K. Zarembo, "Classical / quantum integrability in non-compact sector of AdS/CFT,” JHEP 0410, 060 (2004), hep-th/0410105.

[22] S. Frolov and A. A. Tseytlin, "Semiclassical quantization of rotating superstring in $A d S_{5} \times S^{5}$," JHEP 0206 (2002) 007, hep-th/0204226; "Multi-spin string solutions in $A d S_{5} \times S^{5}$," Nucl. Phys. B 668 (2003) 77, hep-th/0304255; "Quantizing three-spin 
string solution in $A d S_{5} \times S^{5}$," JHEP 0307 (2003) 016, hep-th/0306130; "Rotating string solutions: AdS/CFT duality in non-supersymmetric sectors," Phys. Lett. B $\mathbf{5 7 0}$ (2003) 96, hep-th/0306143.

[23] N. Beisert, J. A. Minahan, M. Staudacher and K. Zarembo, "Stringing spins and spinning strings," JHEP 0309 (2003) 010, hep-th/0306139.

[24] N. Beisert, S. Frolov, M. Staudacher and A. A. Tseytlin, "Precision spectroscopy of AdS/CFT," JHEP 0310 (2003) 037, hep-th/0308117.

[25] R. R. Metsaev, "Type IIB Green-Schwarz superstring in plane wave Ramond-Ramond background," Nucl. Phys. B 625 (2002) 70, hep-th/0112044; R. R. Metsaev and A. A. Tseytlin, "Exactly solvable model of superstring in plane wave Ramond-Ramond background," Phys. Rev. D 65 (2002) 126004, hep-th/0202109.

[26] D. Berenstein, J. M. Maldacena and H. Nastase, "Strings in flat space and pp waves from $N=4$ super Yang Mills," JHEP 0204 (2002) 013, hep-th/0202021.

[27] A. Parnachev and A. V. Ryzhov, "Strings in the near plane wave background and AdS/CFT, " JHEP 0210 (2002) 066, hep-th/0208010.

[28] C. G. Callan, H. K. Lee, T. McLoughlin, J. H. Schwarz, I. Swanson and X. Wu, "Quantizing string theory in $A d S_{5} \times S^{5}$ : Beyond the pp-wave," Nucl. Phys. B 673 (2003) 3, hep-th/0307032; C. G. Callan, T. McLoughlin and I. Swanson, "Holography beyond the Penrose limit," Nucl. Phys. B 694 (2004) 115, hep-th/0404007.

[29] C. G. Callan, T. McLoughlin and I. Swanson, "Higher impurity AdS/CFT correspondence in the near-BMN limit," Nucl. Phys. B 700, 271 (2004), hep-th/0405153.

[30] C. G. Callan, J. Heckman, T. McLoughlin and I. Swanson, "Lattice super Yang-Mills: A virial approach to operator dimensions," Nucl. Phys. B 701, 180 (2004), hep-th/0407096.

[31] T. McLoughlin and I. Swanson, "N-impurity superstring spectra near the pp-wave limit," Nucl. Phys. B 702, 86 (2004), hep-th/0407240.

[32] R. Hernandez and E. Lopez, "Spin chain sigma models with fermions," JHEP 0411, 079 (2004), hep-th/0410022.

[33] N. Beisert, "BMN operators and superconformal symmetry," Nucl. Phys. B 659 (2003) 79, hep-th/0211032.

[34] A. V. Ryzhov and A. A. Tseytlin, "Towards the exact dilatation operator of $\mathcal{N}=4$ super Yang-Mills theory," Nucl. Phys. B 698, 132 (2004), hep-th/0404215.

[35] A. V. Kotikov, L. N. Lipatov, A. I. Onishchenko and V. N. Velizhanin, "Three-loop universal anomalous dimension of the Wilson operators in $\mathcal{N}=4$ SUSY Yang-Mills model," Phys. Lett. B 595 (2004) 521 hep-th/0404092. 
[36] S. Moch, J. A. M. Vermaseren and A. Vogt, "The three-loop splitting functions in QCD: The non-singlet case," Nucl. Phys. B 688 (2004) 101, hep-ph/0403192.

[37] F. A. Dolan and H. Osborn, "Conformal four point functions and the operator product expansion," Nucl. Phys. B 599 (2001) 459, hep-th/0011040.

[38] G. Arutyunov, B. Eden, A. C. Petkou and E. Sokatchev, "Exceptional non-renormalization properties and OPE analysis of chiral four-point functions in $\mathcal{N}=4$ SYM(4)," Nucl. Phys. B 620 (2002) 380, hep-th/0103230.

[39] J. M. Maldacena, "The large $N$ limit of superconformal field theories and supergravity," Adv. Theor. Math. Phys. 2 (1998) 231 [Int. J. Theor. Phys. 38 (1999) 1113], hep-th/9711200.

[40] N. Beisert, C. Kristjansen, J. Plefka and M. Staudacher, "BMN gauge theory as a quantum mechanical system," Phys. Lett. B 558 (2003) 229, hep-th/0212269.

[41] L. D. Faddeev, "How Algebraic Bethe Ansatz works for integrable model," hep-th/9605187.

[42] H. Bethe, "Zur Theorie der Metalle. I. Eigenwerte und Eigenfunktionen der Linearen Atomkette," Z. Physik 71 (1931), 205.

[43] M. Staudacher, in preparation.

[44] K. Zarembo, unpublished.

[45] B. Sutherland, "A brief history of the quantum soliton with new results on the quantization of the Toda lattice," Rocky Mtn. J. of Math. 8 (1978) 431.

[46] V. I. Inozemtsev, "Integrable Heisenberg-van Vleck chains with variable range exchange," Phys. Part. Nucl. 34 (2003) 166 [Fiz. Elem. Chast. Atom. Yadra 34 (2003) 332], hep-th/0201001.

[47] M. Lübcke and K. Zarembo, "Finite-size corrections to anomalous dimensions in $N$ = 4 SYM theory," JHEP 0405 (2004) 049, hep-th/0405055.

[48] S. A. Frolov, I. Y. Park and A. A. Tseytlin, "On one-loop correction to energy of spinning strings in $S^{5}$," hep-th/0408187.

[49] B. Eden, in preparation.

[50] N. Beisert, "Spin chain for quantum strings," hep-th/0409054.

[51] I. Swanson, "On the integrability of string theory in $A d S_{5} \times S^{5}$," hep-th/0405172.

[52] S. S. Gubser, I. R. Klebanov and A. M. Polyakov, "A semi-classical limit of the gauge/string correspondence," Nucl. Phys. B 636 (2002) 99, hep-th/0204051.

[53] M. Smedbäck, "Pulsating strings on $A d S_{5} \times S^{5}$," JHEP 0407, 004 (2004), hep-th/0405102. 


\begin{tabular}{|l|ll|l|c|l|}
\hline$\Delta_{0}$ & $L$ & $M$ & $\mathfrak{s u}(2 \mid 3)$ & $\left(n_{1}, \ldots, n_{M}\right)$ & $\left(E_{0}, E_{2}, E_{4}\right)^{P}$ \\
\hline 4 & 3 & 2 & {$[0 ; 1 ; 0,0]$} & $(-1,1)$ & $(6,-12,42)^{+}$ \\
\hline 5 & 4 & 2 & {$[0 ; 1 ; 0,1]$} & $(-1,1)$ & $(4,-6,17)^{-}$ \\
\hline 6 & 5 & 2 & {$[0 ; 1 ; 0,2]$} & $\begin{array}{l}(-1,1) \\
(-2,2)\end{array}$ & $\begin{array}{l}(2.76393,-2.90983,6.1067)^{+} \\
(7.23607,-14.0902,52.3933)^{+}\end{array}$ \\
\hline 7 & 6 & 2 & {$[0 ; 1 ; 0,3]$} & $(-1,1)$ & $\begin{array}{l}\left(2,-\frac{3}{2}, \frac{37}{16}\right)^{-} \\
\left(6,-\frac{21}{2}, \frac{555}{16}\right)^{-}\end{array}$ \\
\hline 7 & 5 & 4 & {$[2 ; 3 ; 0,0]$} & $(-2,-1,1,2)$ & $\left(10,-20, \frac{145}{2}\right)^{-}$ \\
\hline 7.5 & 6 & 3 & {$[1 ; 2 ; 0,2]$} & $\pm(-3,1,2)$ & $(8,-14,49)^{ \pm}$ \\
\hline 8 & 7 & 2 & {$[0 ; 1 ; 0,4]$} & $(-1,1)$ & $(1.50604,-0.830063,0.95726)^{+}$ \\
& & & $(-2,2)$ & $(4.89008,-7.30622,20.9555)^{+}$ \\
& & & $(-3,3)$ & $(7.60388,-14.8637,57.0872)^{+}$ \\
\hline 8 & 6 & 4 & {$[2 ; 3 ; 0,1]$} & $(-2,-1,1,2)$ & $(8,-14,46)^{+}$ \\
\hline 8.5 & 7 & 3 & {$[1 ; 2 ; 0,3]$} & $\pm(-3,1,2)$ & $\left(7,-12, \frac{83}{2}\right)^{ \pm}$ \\
\hline 9 & 8 & 2 & {$[0 ; 1 ; 0,5]$} & $(-1,1)$ & $(1.17157,-0.489592,0.432593)^{-}$ \\
& & & $(-2,2)$ & $\left(4,-5, \frac{49}{4}\right)^{-}$ \\
& & & $(-3,3)$ & $(6.82843,-12.5104,44.3174)^{-}$ \\
\hline 9 & 7 & 4 & {$[2 ; 3 ; 0,2]$} & $(-2,-1,1,2)$ & $(6.39612,-9.3993,27.0234)^{-}$ \\
& & & $(-3,-1,1,3)$ & $(9.10992,-17.1028,63.4254)^{-}$ \\
& & & $(-3,-2,2,3)$ & $(12.494,-24.4979,88.5512)^{-}$ \\
\hline 9.5 & 8 & 3 & {$[1 ; 2 ; 0,4]$} & $\pm(-3,1,2)$ & $\left(6,-\frac{19}{2}, \frac{247}{8}\right)^{ \pm}$ \\
& & & $\pm(-4,1,3)$ & $\left(8,-\frac{29}{2}, \frac{427}{8}\right)^{ \pm}$ \\
\hline
\end{tabular}

Table 1: The three-loop spectrum of the first few excited states of the fermionic subsector $\mathfrak{s u}(1 \mid 1)$ as computed from the explicit solution (3.11). The latter is derived from the perturbative Bethe ansatz. The mode numbers entering the fundamental equation are indicated. The eigenvalues are either rational or algebraic; in the latter case we have given them to five digit precision. In order to compare with the results of direct matrix diagonalization in [14] (see also p.143 of [19]) we have indicated the labels of the corresponding highest weight state of $\mathfrak{s u}(2 \mid 3)$ (no $\mathfrak{s u}(1 \mid 1)$ state is primary in $\mathfrak{s u}(2 \mid 3)$ ). The agreement is perfect, and establishes perturbative factorized scattering in the fermionic sector. Note that the agreement persists up to large magnon densities, e.g. $M=4$ magnons in a length $L=5$ spin chain. $P$ denotes the parity of a state. 


\begin{tabular}{|l|ll|c|l|}
\hline$\Delta_{0}$ & $L$ & $M$ & $\left(n_{1}, \ldots, n_{M}\right)$ & $\left(E_{0}, E_{2}, E_{4}\right)^{P}$ \\
\hline \hline 4 & 2 & 2 & $(-1,1)$ & $(\mathbf{6},-\mathbf{1 2}, \mathbf{4 2})^{+}$ \\
\hline \hline 5 & 3 & 2 & $(-1,1)$ & $(4,-6,17)^{-}$ \\
\hline \hline 6 & 4 & 2 & $(-1,1)$ & $(2.76393,-2.90983,6.1067)^{+}$ \\
& & $(-2,2)$ & $(7.23607,-14.0902,52.3933)^{+}$ \\
\hline 6 & 3 & 3 & $\pm(-2,1,1)$ & $\left(\frac{15}{2},-\frac{225}{16}, \frac{3195}{64}\right)^{ \pm}$ \\
\hline 6 & 2 & 4 & $(-1,-1,1,1)$ & $\left(\frac{\mathbf{2 5}}{3},-\frac{925}{54}, \frac{241325}{3888}\right)^{+}$ \\
\hline \hline 7 & 5 & 2 & $(-1,1)$ & $\left(2,-\frac{3}{2}, \frac{37}{16}\right)^{-}$ \\
& & $(-2,2)$ & $\left(6,-\frac{21}{2}, \frac{555}{16}\right)^{-}$ \\
\hline 7 & 4 & 3 & $\pm(-2,1,1)$ & $\left(6,-\frac{21}{2}, 36\right)^{ \pm}$ \\
\hline 7 & 3 & 4 & $(-1,-1,1,1)$ & $\left(6,-\frac{39}{4}, \frac{957}{32}\right)^{-}$ \\
\hline \hline 8 & 6 & 2 & $(-1,1)$ & $(1.50604,-0.830063,0.95726)^{+}$ \\
& & & $(-2,2)$ & $(4.89008,-7.30622,20.9555)^{+}$ \\
& & & $(-3,3)$ & $(7.60388,-14.8637,57.0872)^{+}$ \\
\hline 8 & 5 & 3 & $\pm(-2,1,1)$ & $(4.72931,-7.01464,21.1993)^{ \pm}$ \\
& & & $\pm(-3,1,2)$ & $(7.77069,-14.4229,52.8944)^{ \pm}$ \\
\hline 8 & 4 & 4 & $(-1,-1,1,1)$ & $(4.38277,-5.25026,12.58394)^{+}$ \\
& & & $(-2,-1,1,2)$ & $(8.35923,-16.0680,59.3810)^{+}$ \\
& & & $(-2,-2,2,2)$ & $(11.5913,-23.1031,83.6199)^{+}$ \\
& & & $\pm(1,1,1,1)$ & $\left(\frac{23}{3},-\frac{1331}{108}, \frac{76973}{1944}\right)^{ \pm}$ \\
\hline 8 & 3 & 5 & $\pm(-2,-1,1,1,1)$ & $\left(\frac{35}{4},-\frac{18865}{1152}, \frac{1068515}{18432}\right)^{ \pm}$ \\
\hline 8 & 2 & 6 & $(-1,-1,-1,1,1,1)$ & $\left(\frac{49}{5},-\frac{45619}{2250}, \frac{300642097}{4050000}\right)^{+}$ \\
\hline
\end{tabular}

Table 2: The three-loop spectrum of the first few highest weight states of the derivative subsector $\mathfrak{s l}(2)$ as derived from the perturbative Bethe ansatz. The mode numbers entering the fundamental equation are indicated. The eigenvalues are either rational or algebraic; in the latter case we have given them to five digit precision. The one-loop spectrum was found by direct matrix diagonalization in [13] (see also p.84 of [19]). At the time of writing this spectrum cannot be checked against direct diagonalization since the $\mathfrak{s l}(2)$ dilatation operator is not yet known beyond one loop. The bold-faced values with $L=2$ correspond to twist-two operators and agree perfectly with results of Kotikov, Lipatov, Onishchenko and Velizhanin. The values of two-magnon states $M=2$ were previously known from superconformal invariance. The three-loop results for the cases $L=2,3$ and $M=2$ were first predicted in [2] and confirmed in a field theory computation in [18]. The two- and three loop anomalous dimensions of all twist- $L$ operators with $L>2$ and $M>2$ are new predictions. The parity of a state is denoted by $P$. The case $M=4, L=4$ with mode numbers $\pm(1,1,1,1)$ is interesting as it is the simplest example of a paired state in $\mathfrak{s l}(2)$ where all Bethe roots are either strictly positive or strictly negative real. Such states with "winding number" were considered, in the thermodynamic limit, in [53, 21], and it is reassuring to find them in the finite spin chain. 